\title{
Random-Field Ising Models of Hysteresis
}

\author{
James P. Sethna \\ Laboratory of Atomic and Solid State Physics (LASSP), Clark Hall, Cornell University, Ithaca, NY 14853-2501, \\ USA
}

Karin A. Dahmen

Physics Department, University of Illinois at Urbana/Champaign, 1110 West Green Street, Urbana, IL 61801-3080, USA

Olga Perković

URS Corporation, 600 Montgomery Street, 26th floor, San Francisco, CA 94111-2727, USA

\begin{abstract}
This is a review article of our work on hysteresis, avalanches, and criticality. We provide an extensive introduction to scaling and renormalization-group ideas, and discuss analytical and numerical results for size distributions, correlation functions, magnetization, avalanche durations and average avalanche shapes, and power spectra. We focus here on applications to magnetic Barkhausen noise, and briefly discuss non-magnetic systems with hysteresis and avalanches.
\end{abstract}

\section{Contents}

I. Introduction

II. Models

III. The Renormalization Group and Scaling

IV. Critical Exponents, Scaling Functions

V. Measuring Exponents and Scaling Functions A. Magnetization Curves

B. Avalanche Size Distribution

C. Avalanche Correlations

D. Avalanche Duration Measurement

E. Energy Spectrum

F. Tables of Results

VI. Exponent relations

A. Exponent equalities

B. Two violations of hyperscaling

Exponent inequalities

VII. Finite Sweeprate

VIII. Subloops and History Induced Critical Behavior 18

A. Return-Point Memorv

B. Critical Behavior in Subloops

C. Demagnetization Curves

IX. Real Experiments

A. Magnetic hysteresis loops

B. Disorder effects on Barkhausen Noise

C. Imaging magnetic avalanches and states

D. Random Bonds and Random Anisotropv

E. Other dvnamics at high sweep rates

F. Nonmagnetic noisv hysteretic systems

1. Svstems with avalanches

2. Martensites

3. Liquid Helium in Nuclepore

4. Superconductors

5. Prewetting on a disordered substrate

X. Unsolved problems

Acknowledgments

1

3

5

7
A. Derivation of the scaling forms and corrections

26

References

29

Our group has devoted several years to the study of the dynamics of a simple model, the random-field Ising model at zero temperature. We do clever simulations involving the dynamics of billions of domains (figure1), and we develop sophisticated analytical methods for extracting and explaining the properties of this model. We do this because we believe our model - despite its dramatically simplified nature - may well describe the properties of Barkhausen noise in many real physical systems.

We present here the results of our simulations and analysis, together with an explanation of why we believe they should (or could) describe real experiments. Our arguments for the applicability of our model are based on renormalization group and scaling theories, first developed to study continuous phase transitions in equilibrium systems. To a large extent, these theories can be seen as the underlying reason why many if not most theories of nature apply to the real world, and (more specifically) why different magnets share common features in their dynamics despite having microscopically rather different morphologies and energetics.

Why is the noise in magnets interesting? As one applies an external field to most magnetic materials, the magnetization changes through the nucleation and motion of domain walls. This motion is not smooth, and the resulting jumps in the net magnetization is termed Barkhausen noise. These jumps, corresponding to the reorganization (or avalanche) of a region of spins, usually span many decades in size: there will be many small avalanches of spins, and fewer and fewer avalanches of larger and larger sizes (what we call crackling noise). Indeed, the probability $D$ of having a jump or avalanche 


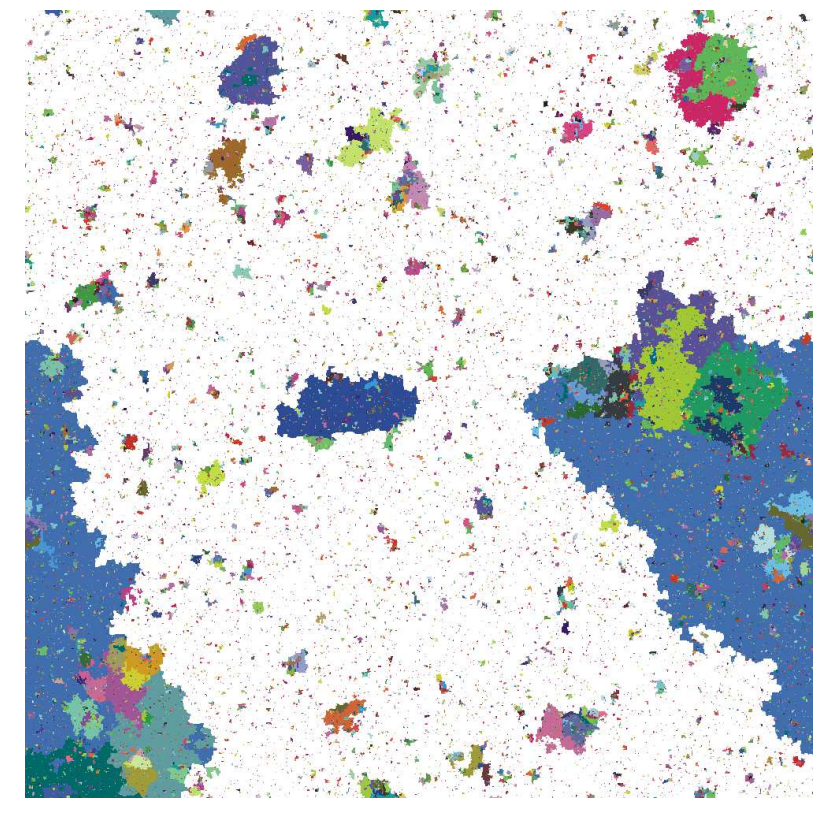

FIG. 1 Cross section of all avalanches in a billion-spin simulation at the critical disorder (1). The white background is the infinite, spanning avalanche, which would not exist above $R_{c}$.

of a given size $S$ often decreases as a power law ${ }^{1}$ in the size of the avalanche $D(S) \sim S^{-\tau}$ for a significant range of sizes (figures 2 and 3). Simple behavior (like a power law) emerging out of complicated microscopic dynamics is a good sign that something needs explanation! Power laws in particular are the defining signature of continuous phase transitions, and a large fraction of the statistical physics community focuses on problems involving these power laws: a new class of systems to study is exciting. Finally, similar power laws for crackling noise emerge in many other systems (1), from flux-line avalanches in superconductors, to crumpling paper (4), to earthquakes (the Gutenberg-Richter law). Magnetic Barkhausen noise forms a manageable experimental prototype of a whole class of behavior.

Why should crackling noise in magnets be comprehensible? Very small jumps in the magnetization, corresponding to avalanches on scales comparable to the microstructure, will certainly depend upon the details of the individual magnetic material. Large jumps, spanning the entire sample, will depend upon the sample geometry. Indeed, these extremes in sizes are not regions in which simple power laws should be observed experimentally. But these extremes are separated by many powers of ten in a typical experiment. Just as the complex mi-

\footnotetext{
${ }^{1}$ In our model, this law is obtained only at the critical field and at the critical disorder (figure 3 integrating over field yields a different power law $D_{\text {int }}(S) \sim S^{-(\tau+\sigma \beta \delta)}$ at the critical disorder, see equation 10
}

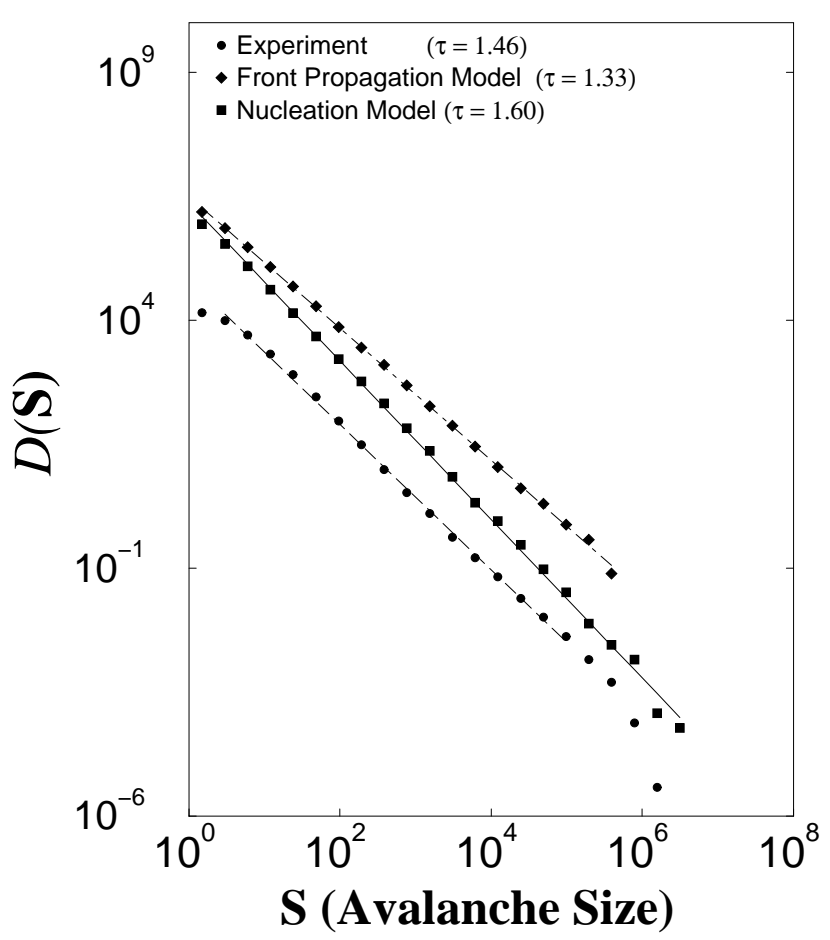

FIG. 2 Avalanche size distributions (2). The figure shows the number $D(S)$ of avalanches of size $S$ measured in an experiment (2) measuring near the center of the hysteresis loop, our (nucleation) model near $R_{c}$, and a related front propagation model. Notice the straight lines on a log-log plot indicate power-law behaviors $D(S) \sim S^{-\tau}$.

croscopic properties of the molecules in a fluid only affect the viscosity and density on long length scales, so also one might expect the complicated microstructure in a magnet could be subsumed into a few constants when dealing with events of sizes much larger than the microstructure. The smooth power-law behavior suggests exactly this: something simple is happening on intermediate scales, independent of either the microscopic or macroscopic details.

What should be explicable? How ambitious can we be in our expectations of a successful theory? As we shall explain in this review, we cannot expect to be able to tell when and where to expect an avalanche. (Our tools hence will likely not be effective at predicting large earthquakes.) However, a successful theory should predict statistical averages of almost any quantity that is dominated by events on large length and time scales, up to certain overall parameter-dependent scales (analogous to viscosity and density for fluids). For example, the probability $D(S, T, L, W, R, H)$ in our theory of having an avalanche of size $S$, duration $T$, long axis $L$, short axis $W$, at disorder $R$ and external field $H$ should be predicted by the theory, up to an overall size scale $S_{s}$, time scale $T_{s}$, field scale $H_{s}$ and offset $H_{c}$, and disorder scale $R_{s}$ and offset $R_{c}$. Indeed, we shall see that functions like these take 


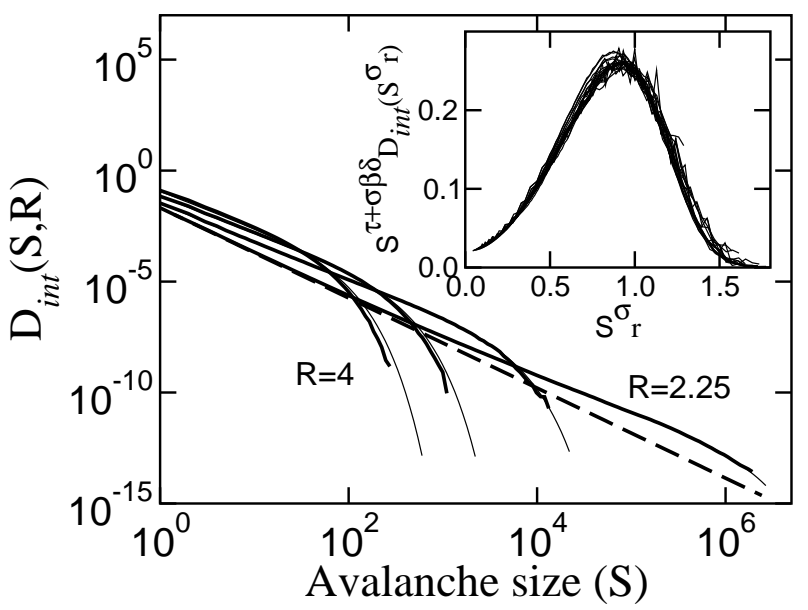

FIG. 3 Avalanche size distributions for our model (3), integrated over the hysteresis loop, for a range of disorders above $R_{c}=2.16$ : at $R_{c}$ one gets a power law (the straight dashed line) $D_{\text {int }}\left(S, R_{c}\right) \propto s^{-(\tau+\sigma \beta \delta)}$. The inset shows a scaling collapse of the data (equation 10). In the main figure, the thin lines show the scaling function prediction for the associated curves. The true power-law behavior only emerges very close to $R_{c}$, but the universal scaling function governs a range of disorders spanning a factor of two above $R_{c}$. Notice also that we get six orders of magnitude of scaling at $5 \%$ above $R_{c}$, without any self-organization.

scaling forms

$$
\begin{aligned}
& D_{\text {multi }}(S, T, L, W, R, H) \\
& \quad=D_{s} S^{-(\tau+\sigma \nu z)} \mathcal{D}_{\text {multi }}\left(\frac{S}{S_{s}} r^{1 / \sigma}, \frac{T}{T_{s}} r^{z \nu}, \frac{L}{W}, \frac{h}{r^{\beta \delta}}\right)(1)
\end{aligned}
$$

where $r=\frac{R-R_{c}}{R_{s}}, h=\frac{H-H_{c}}{H_{s}}, D_{s}$ is a normalization factor, and $\tau+\sigma \nu z$ is another universal critical exponent. We shall derive these scaling forms from self-similarity or scale invariance: the avalanche dynamics is inherently the same when observed (say) at microns and hundreds of microns. This scale invariance allows us to write functions of $N$ variables as power laws times functions of $N-1$ variables; hence, functions of one variable become pure power laws, as for $D(S)$ above. What makes these theories predictive is that both the power law $\tau$ and the entire multivariable function $\mathcal{D}_{\text {multi }}$ are universal: they are independent of the microscopic model (within large classes of systems). If your theory is in the same universality class as the experiment, it will predict all critical exponents and scaling functions.

There are three important qualifications about these predictions.

1. Analytic corrections. The various scales $S_{s}, T_{s}$, $R_{c}, H_{c}, \ldots$ will not be constants, but will depend smoothly and analytically on the control parameters in a given system. This is a serious issue, as the function we are describing ( $D_{\text {multi }}$ above) will typically itself be a smooth and analytic function of its parameters except at a phase transition where something qualitatively changes in the behavior of the system. Indeed, it is precisely at these phase transitions when events of all scales arise, where we expect our theory to apply. These analytic corrections usually become less and less important as we confine our attention to large, slow events near the transition.

2. Universality Classes. The power of the theory rests in the prediction that quite different experimental systems, or a theoretical model and an experiment, may be in the same universality class. We shall see that universality classes are studied by considering a space of possible systems: if two systems flow towards the same fixed point as one coarse-grains to larger scales, then their long length scale behaviors must agree. In many cases, and for magnetic noise in particular, there can be more than one candidate theory. Our model which incorporates short-range interactions and nucleation of new domains has competitors which allow only interface depinning $(\underline{5} ; \underline{6})$ and which incorporate long-range fields in a mean-field fashion (7; 8 ; 9 ; 10; 11; 12). For many properties, the predictions of the various theories are not strikingly different: careful experiments may be needed to distinguish between the rival theories if it is not obvious whether nucleation or long-range interactions are relevant in a given experiment.

3. Dynamic criticality. The static, equal-time properties of these models have historically been less fussy than the time-dependent behavior. More specifically, there will often be several dynamic universality classes for each static universality class, at least in the well-studied cases of equilibrium continuous phase transitions. We will see clear evidence that our theories are not predicting the dynamical behavior within the avalanches properly, but that the various theories are rather successful at describing the distributions of avalanches and other static quantities.

\section{MODELS}

Several variations of the zero temperature random field Ising model have been proposed to explain the power laws in Barkhausen noise. They are differentiated on the basis of the presence of long range forces, and the details of the dynamics. Our summary follows reference (13).

The model is composed of a large number of 'spins' $s_{i}= \pm 1$ on a cubic lattice (square lattice in two dimensions, hypercubic for dimensions $D>3$ ). These spins represent domains or small regions of the material. The model is subject to an external field $H$, which varies 


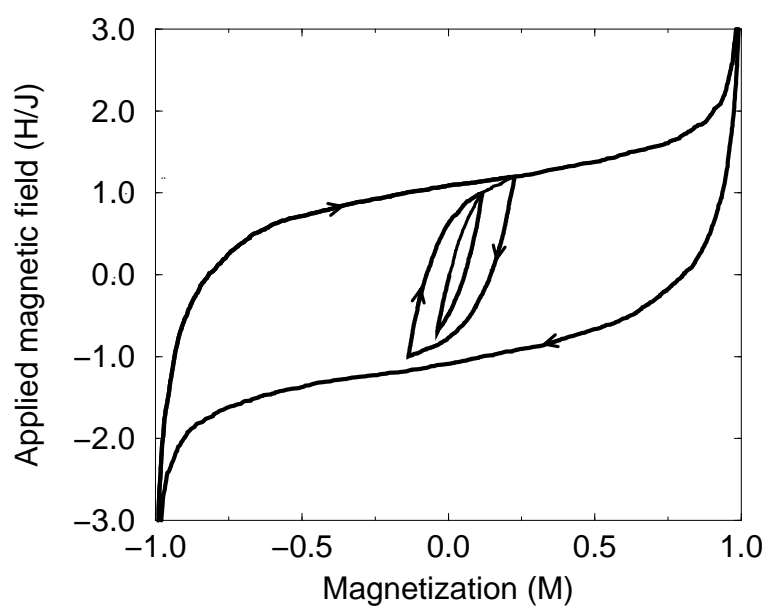

FIG. 4 Hysteresis loop and subloops. The magnetization in our model (for $R>R_{c}$ ), as the external field $H$ is ramped up and down. Our focus will primarily be on the upper, outer loop as the external field is ramped from $-\infty$ to $\infty$.

slowly in time, ${ }^{2}$ usually ramping upward from $H=-\infty$ to $H=\infty$. The value of a spin $s_{i}$ represents whether that domain is aligned $(+1)$ or anti-aligned $(-1)$ with the final $H=+\infty$. The energy function, or Hamiltonian, for the models is

$$
\begin{aligned}
\mathcal{H}= & -\sum_{\mathrm{nn}} J_{\mathrm{nn}} s_{i} s_{j}-\sum_{i} H s_{i}-\sum_{i} h_{i} s_{i} \\
& +\sum_{i} \frac{J_{\mathrm{inf}}}{N} s_{i}-\sum_{\{i, j\}} J_{\text {dipole }} \frac{3 \cos ^{2}\left(\theta_{i j}\right)-1}{r_{i j}^{3}} s_{i} s_{j} .
\end{aligned}
$$

Here $J_{\mathrm{nn}}$ is the strength of the ferromagnetic nearest neighbor interactions, $h_{i}$ is a random field representing the effects of compositional and morphological disorder, $J_{\text {inf }}$ is the strength of an infinite range demagnetizing field, (8) and $\theta_{i j}$ is the angle between the positive spin direction and the difference vector between lattice positions $i$ and $j$, and $J_{\text {dipole }}$ is the strength of the dipoledipole interactions. The critical exponents of the power laws are independent of the particular choice of random field distributions $\rho\left(h_{i}\right)$ for a large variety of distributions. We use a Gaussian distribution of random fields, with zero mean and standard deviation $R$. (When we refer to the strength of the disorder, we are referring to the width, $R$, of the random field distribution. $)^{3}$

\footnotetext{
2 Except when otherwise mentioned, the field varies slowly enough that each avalanche finishes before the field changes.

${ }^{3}$ In this review we focus specifically on zero temperature models; there are also finite temperature studies especially for pure systems (14; 15) and micromagnetic systems (16), interface depinning models in the presence of temperature $(17 ; 18)$ and hysteresis due to driven interfaces at finite temperature $(19 ; 20 ; 21)$.
}

Two different dynamics have been considered. The first is a front propagation dynamics in which a spin on the edge of an existing front flips as soon as it would decrease the energy to do so, introduced by Ji and Robbins (5). Spins with no flipped neighbors cannot flip even if it would be energetically favorable. Second is the dynamics we use (23), which includes domain nucleation. Any spin can flip when it becomes energetically favorable to do so. In both cases, spins flip in shells - all spins which can flip at time $t$ flip, then all of their newly flippable neighbors flip at time $t+1$, causing an avalanche. The number of spins flipped in each shell gives the time series of the avalanche (figure 5), whose irregular fluctuations and near halts are typical also of experimental time series.

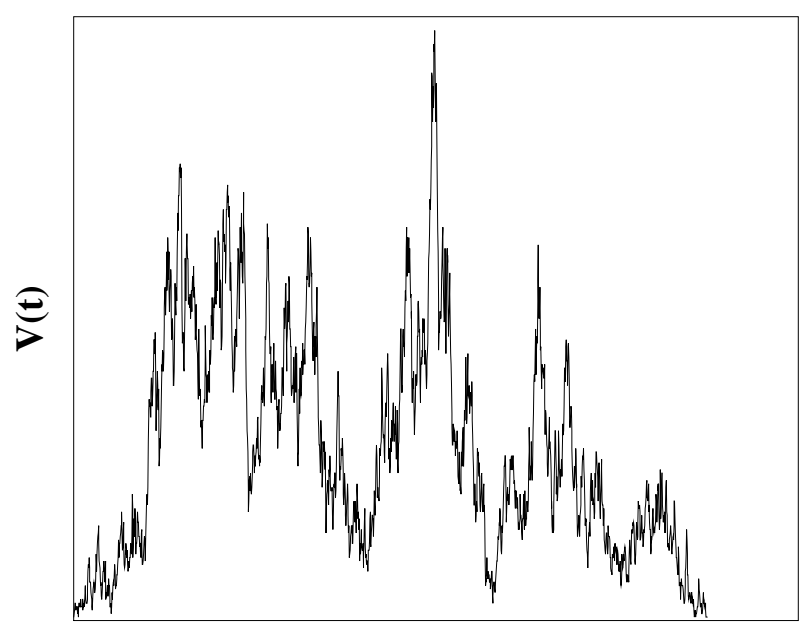

$\mathbf{t}$

FIG. 5 Typical avalanche time series. (13) Voltage (number of domains flipped) pulse during a single large avalanche (arbitrary units). Notice how the avalanche almost stops several times: if the forcing were slightly smaller, this large avalanche would have broken up into two or three smaller ones. The fact that the forcing is just large enough to on average keep the avalanche growing is the cause of the selfsimilarity: on average a partial avalanche of size $S$ will trigger one other on size $S$.

Depending on which terms are included in the Hamiltonian, the behavior appears to fall into three different universality classes.

1. Front propagation model (5) . The front propagation model has a critical field $H_{c}$ for a range of disorders $R$. As the field approaches $H_{c}$, larger and larger avalanches arise. As one approaches $H_{c}$ from below these avalanches develop a power-law

We are currently working on hysteresis at finite temperatures with randomness and nucleation (22). 
distribution in sizes with a cutoff which diverges at $H_{c}$. Above $H_{c}$ the entire front is depinned, and the front moves forward with an inhomogeneous velocity which begins jerky on all length and time scales (echoing the avalanches below $H_{c}$ ) but which becomes smoother and more uniform at large $H$.

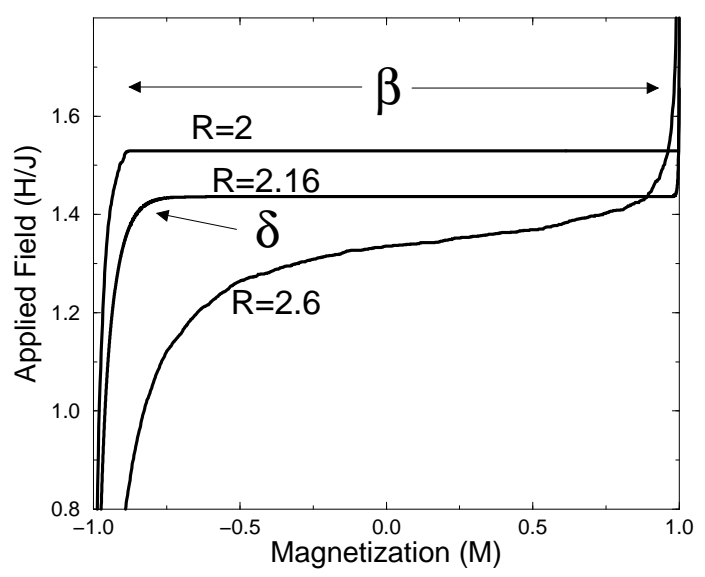

FIG. 6 Infinite avalanche, jump in $M(H)$ for $R<R_{c}$.

2. Our model (nucleation allowed, Figure 4). For our model where domain nucleation is allowed and only nearest neighbor interactions are included, there is a continuous transition at a critical point with disorder $R_{c}$ and external field $H_{c}$. At very large disorder, the couplings between spins $J_{\mathrm{nn}}$ are a small perturbation and each spin $s_{i}$ flips roughly when the external field $H(t)$ is equal and opposite to the local random field $h_{i}$. For all disorders above $R_{c}$ this qualitative behavior persists: all avalanches are finite, dying out after a number of spin flips that does not depend on the number of spins $N$, yielding a smooth hysteresis loop (figure 6 ). For very small disorder, an early spin will trigger almost the entire system to flip over. This too extends qualitatively to all disorders below $R_{c}$, where finite fraction of the spins in the system (even as the system size becomes large) flip in a single event, the infinite or spanning avalanche. The critical disorder $R_{c}$ is defined as the disorder at which the infinite avalanche first arises, and where a jump in the magnetization per spin $m(H)$ first arises in the limit of an infinite system. ${ }^{4}$ Figure 1 shows the avalanches in a cross-section of our system at the critical disorder: the white background is the spanning, or infinite

\footnotetext{
${ }^{4}$ Each avalanche produces a jump in the magnetization $\Delta M$, but all finite avalanches produce jumps in the magnetization per spin $\frac{\Delta M}{N}$ which vanish as the number of spins $N$ diverges.
}

avalanche. ${ }^{5}$ One does not need to be exactly at $R_{c}$ to observe power laws in our model: one gets a large region of power-law scaling rather far from the critical point (figure 3 and reference (3)).

3. Infinite Range and Dipolar Fields. For disorders below $R_{c}$, or when domain nucleation is not allowed, the addition of an infinite-range demagnetizing field ${ }^{6}$ (8) can self-organize the system to a different critical behavior. (Self-organization means that the system naturally sits at a critical point, without having to tune any parameters.) The infinite-ranged interaction is sometimes introduced to mimic the effects of the boundaries of materials with dipolar, or other long-ranged interactions (10); these interactions also self-organize the model to the critical point (9). Our use of the term "infinite-range models" perhaps obscures the clear physical origin of this universality class of models.

Zapperi et al. (10) argue that the addition of dipoledipole interactions to the model lowers the upper critical dimension to three and produces meanfield exponents in three dimensions. Since large mean-field simulations are much easier than large simulations with dipole-dipole interactions, we will give results from mean-field simulations in this paper. Dipolar interactions without an infinite-range term were explored by Magni (24) in two dimensions, who found labyrinthine patterns and hysteresis loops similar to those seen in garnet films.

The infinite-range model is apparently also equivalent to the rather successful single-degree of freedom ABBM models (10; 25; 26).

Unless we specify otherwise, we will focus on our model, allowing nucleation with parameters $J_{\mathrm{nn}}, H$, and $h_{i}$ but without long-range interactions $\left(J_{\mathrm{inf}}=J_{\text {dipole }}=\right.$ $0)$.

\section{THE RENORMALIZATION GROUP AND SCALING}

To study crackling noise, we use renormalization-group tools developed in the study of continuous phase transitions. ${ }^{7}$ The word renormalization has roots in the study

\footnotetext{
${ }^{5}$ It may seem as if the spanning avalanche is occupying quite a large fraction of the volume. As the system size increases, the "holes" formed by the finite avalanches (and other spanning avalanches) will gradually become larger and larger fractions of the total, and the spanning avalanche at $R_{c}$ should grow with the number of spins $N$ to a power below one.

6 This is not the same as a mean-field model, because the model contains nearest neighbor interactions along with the infinite range interactions.

7 This section and the next follow closely the presentation in reference (1). References to the broader literature may be found there.
} 
of quantum electrodynamics, where the effective charge changes in size (norm) as a function of length scale. The word group refers to the family of coarse-graining operations basic to the method: the group product is composition (coarsening repeatedly). The name is unfortunate, however, as the basic coarse-graining operation does not have an inverse, and thus the renormalization group does not have the mathematical structure of a group.

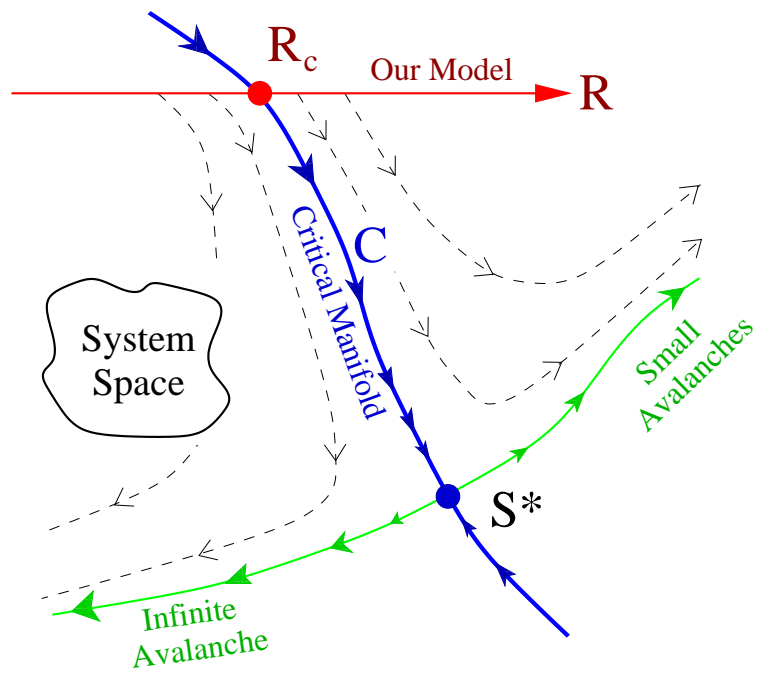

FIG. 7 Renormalization-group flows (1). The renormalization-group uses coarse-graining to longer length scales to produce a mapping from the space of physical systems into itself. Consider the space of all possible systems exhibiting magnetic hysteresis (including, in our imagination, both real models and experimental systems). Each model can be coarse-grained, removing some fraction of the microscopic domains and introducing more complex dynamical rules so that the remaining domains still flip over at the same external fields. This defines a mapping of our space of models into itself. A fixed point $\mathbf{S}^{*}$ in this space will be self-similar: because it maps into itself upon coarse-graining, it must have the same behavior on different length scales. Points that flow into $\mathbf{S}^{*}$ under coarse-graining share this self-similar behavior on sufficiently long length scales: they all share the same universality class.

The renormalization group studies the way the space of all physical systems maps into itself under coarsegraining (see figure 7). The coarse-graining operation shrinks the system, and removes degrees of freedom on short length scales. Under coarse-graining, we often find a fixed point $\mathbf{S}^{*}$ : many different models flow into the fixed point and hence share long-wavelength properties. To get a schematic view of coarse-graining, look at figures 1 and 8 the $1000^{3}$ cross section looks (statistically) like the $100^{3}$ section if you blur your eyes by a factor of 10 .

Much of the mathematical complexity of this field involves finding analytical tools for computing the flow diagram in figure 7 Using methods developed to study thermodynamical phase transitions and the depinning of charge-density waves, we can calculate for our model the flows for systems in dimensions close to six (the so-called $\epsilon$ expansion, where $\epsilon=6-D, D$ being the dimension of the system). Interpolating between dimensions may seem a surprising thing to do. In our system it gives rather good predictions even in three dimensions (i.e., $D=3$ ), but it's hard work, and we won't discuss it here. Nor will we discuss real-space renormalization-group methods or series expansion methods. We focus on the relatively simple task of using the renormalization group to justify and explain the universality, self-similarity, and scaling observed in nature.

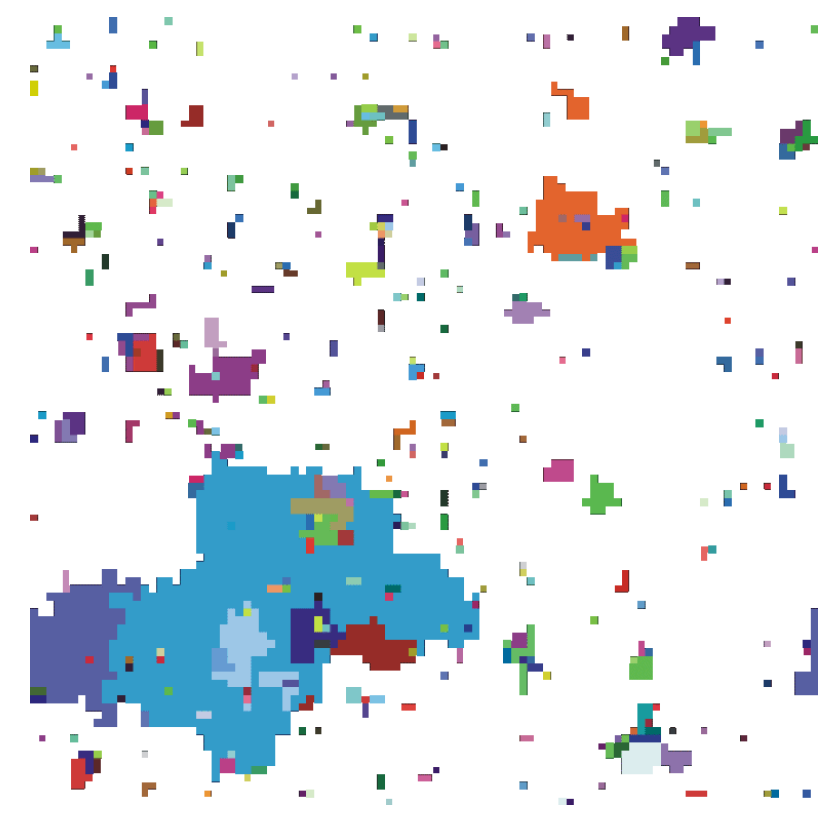

FIG. 8 Cross section of all avalanches in a million-spin simulation at the critical disorder (1). Compare with the much larger simulation in figure 1

Consider the "system space" for disordered magnets. There is a separate dimension in system space for each possible parameter in a theoretical model (disorder, coupling, next-neighbor coupling, dipolar fields etc.) or in an experiment (temperature, annealing time, chemical composition ...). Coarse-graining, however one implements it, gives a mapping from system space into itself: shrinking the system and ignoring the shortest length scales yields a new physical system with identical long-distance physics, but with different (renormalized) values of the parameters. We've abstracted the problem of understanding crackling noise in magnets into understanding a dynamical system acting on the space of all dynamical systems.

Figure 7 represents a two-dimensional cross section of this infinite-dimensional system space. We've chosen the cross section to include our model: as we vary the disorder $R$, our model sweeps out a straight line in system space. The cross section also includes a fixed point $\mathbf{S}^{*}$, which maps into itself under coarse-graining. The system $\mathbf{S}^{*}$ looks the same on all length and time scales, 
because it coarse-grains into itself. We can picture the cross section of figure 7 either as a plane in system space (in which case the arrows and flows depict projections, since in general the real flows will point somewhat out of the plane), or as the curved manifold swept out by our one-parameter model as we coarse grain (in which case the flows above our model and below the horizontal curved line in figure $\mathbf{7}$ s should be ignored).

The flow near $\mathbf{S}^{*}$ has one unstable direction, leading outward along the horizontal curve (the unstable manifold). In system space, there is a surface of points $\mathbf{C}$ which flow into $\mathbf{S}^{*}$ under coarse-graining. Because $\mathbf{S}^{*}$ has only one unstable direction, $\mathbf{C}$ divides system space into two phases. To the left of $\mathbf{C}$, the systems will have one large, system-spanning avalanche (a snapping noise). To the right of $\mathbf{C}$, all avalanches are finite and under coarse-graining they all become small (popping noise). Our model, as it crosses $\mathbf{C}$ at the value $R_{c}$, goes through a phase transition.

Our model at $R_{c}$ is not self-similar on the shortest length scales (where the square lattice of domains still is important), but because it flows into $\mathbf{S}^{*}$ as we coarsegrain we deduce that it is self-similar on long length scales. Some phase transitions, like ice melting into water, are abrupt and don't exhibit self-similarity. Continuous phase transitions like ours almost always have self-similar fluctuations on long length scales. Also, we must note that our model at $R_{c}$ will have the same selfsimilar structure as $\mathbf{S}^{*}$ does. Indeed, any experimental or theoretical model lying on the critical surface $\mathbf{C}$ will share the same long-wavelength critical behavior. This is the fundamental explanation for universality.

The flows in system space can vary from one class of problems to another: the system space for front propagation models (figure 9a) will have a different flow, and its fixed point will have different scaling behavior (yielding a different universality class). In some cases, a fixed point will attract all the systems in its vicinity (figure 10). Usually at such attracting fixed points the fluctuations become unimportant at long length scales: the NavierStokes equation for fluids described earlier can be viewed as a stable fixed point. The coarse-graining process, averaging over many degrees of freedom, naturally smooths out fluctuations, if they aren't amplified near a critical point by the unstable direction. Fluctuations can remain important when a system has random noise in a conserved property, so that fluctuations can only die away by diffusion: in these cases, the whole phase will have self-similar fluctuations, leading to generic scale invariance.

Sometimes, even when the system space has an unstable direction like in figure $\mathbf{7}$ the observed behavior always has avalanches of all scales. This can occur simply because the physical system averages over a range of model parameters (i.e., averaging over a range of $R$ including $R_{c}$ in figure (7). For example, this can occur by the sweeping of a parameter slowly in time, or varying it gradually in space either deliberately or through

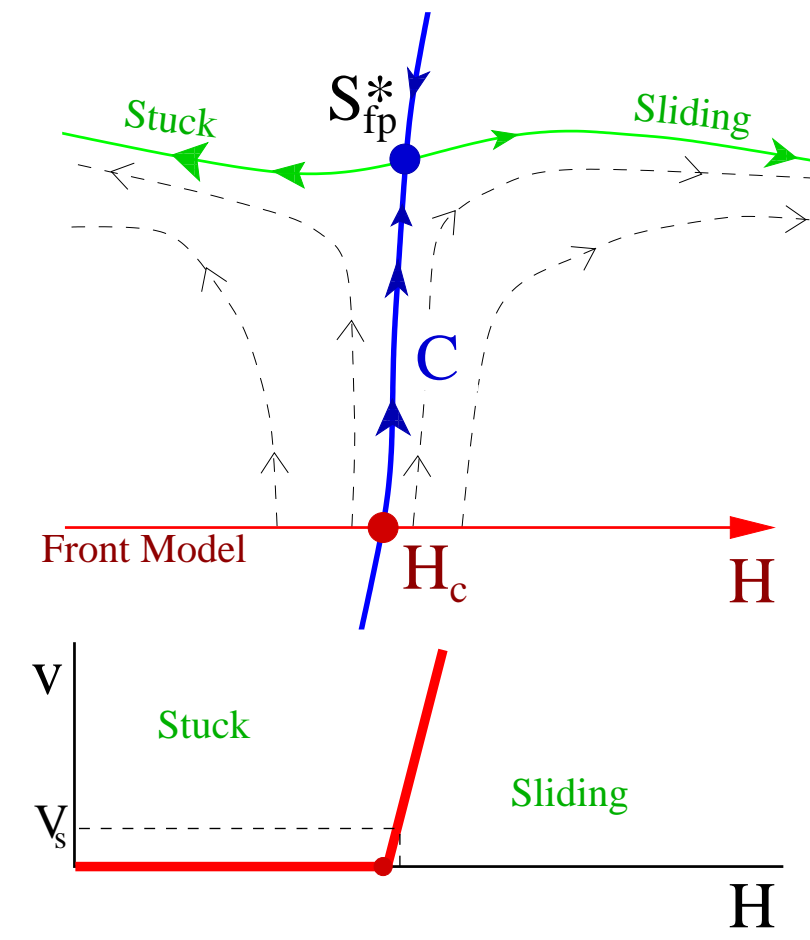

FIG. 9 Flows for a front-propagation model (1). The front propagation model has a critical field $H_{c}$ at which the front changes from a pinned to sliding state. (a) Coarsegraining defines a flow on the space of depinning models. The fixed point $\mathbf{S}^{*}{ }_{f p}$ will have a different local flow field from other renormalization-group fixed points, yielding its own universality class of critical exponents and scaling functions. The critical manifold $\mathbf{C}$, consisting of models which flow into $\mathbf{S}_{f p}{ }_{f p}$, separates the stuck fronts from those which slide forward with an average velocity $v(H)$. (b) The velocity varies with the external force as a power law $v(H) \sim\left(H-H_{c}\right)^{\beta}$. Clever experiments, or long-range fields, can act to control not the external field, but the net magnetization: changing the magnetization slowly sets $v \approx 0$, thus self-tuning $H \approx H_{c}$. This is one example of self-organized criticality.

large-scale inhomogeneities.

One can also have self-organized criticality, where the system is controlled so that it naturally sits on the critical surface. Self-organization to the critical point can occur via many mechanisms. In the front propagation models with long-range fields (figure 9b), upon an increment of external field $H$ the front will move forward until the long-range demagnetization force balances the increment: the net external field given by the sum organizes itself to precisely the critical depinning field for the front.

\section{CRITICAL EXPONENTS, SCALING FUNCTIONS}

The renormalization group is the theoretical underpinning for understanding why universality and self- 


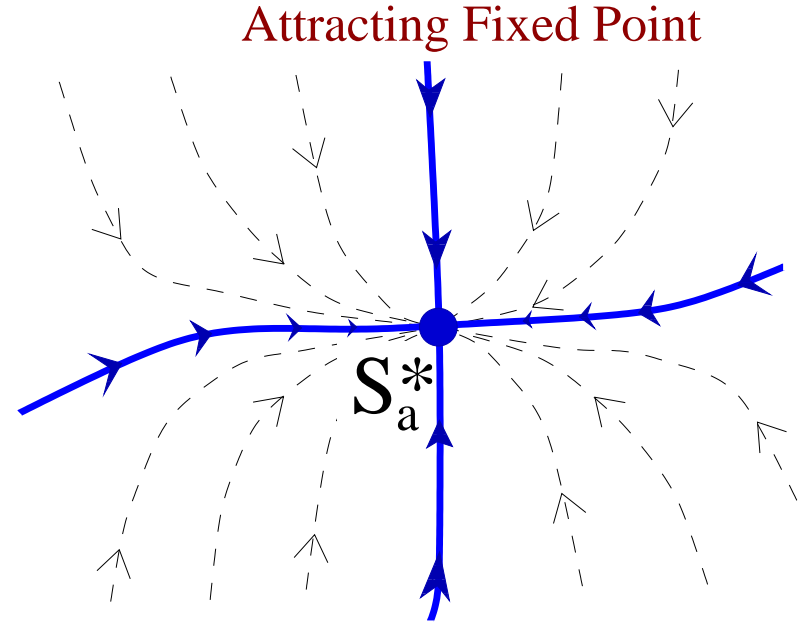

FIG. 10 Attracting fixed point (1). Often there will be fixed points that attract in all directions. These fixed points describe phases rather than phase transitions. Most phases are rather simple, with fluctuations that die away on long length scales. When fluctuations remain important, they will exhibit self-similarity and power laws called generic scale invariance.

similarity occur. ${ }^{8}$ Once we grant that different systems should sometimes share long-distance properties, though, we can quite easily derive some powerful predictions.

To take a tangible example, lets consider the relation between the duration of an avalanche and its size. If we look at all avalanches of a certain duration $T$ in an experiment, they will have a distribution of sizes $S$ around some average $\langle S\rangle_{\exp }(T)$. If we look at a theoretical model, it will have a corresponding average size $\langle S\rangle_{\mathrm{th}}(T)$. If our model describes the experiment, these functions must be essentially the same at large $S$ and large $T$. We must allow for the fact that the experimental units of time and size will be different from the ones in our model: the best we can hope for is that

$$
\langle S\rangle_{\exp }(T)=A\langle S\rangle_{\mathrm{th}}(T / B),
$$

for some rescaling factors $A$ and $B$.

Now, instead of comparing to experiment, we can compare our model to itself on a slightly larger time scale. If the time scale is expanded by a small factor $B=1 /(1-\delta)$, then the rescaling of the size will also be small, say $1+a$. Hence

$$
\langle S\rangle(T)=(1+a \delta)\langle S\rangle((1-\delta) T) .
$$

Making $\delta$ very small yields the simple relation $a\langle S\rangle=$ $T d\langle S\rangle / d T$, which can be solved to give the power law relation $\langle S\rangle(T)=S_{0} T^{a}$. The exponent $a$ is called a critical exponent, and is a universal prediction of a given theory.

\footnotetext{
8 This section also follows closely the presentation in reference (1).
}

(That means that if the theory correctly describes an experiment, the critical exponents will agree.) In our work, we write the exponent $a$ relating time to size in terms of three other critical exponents, $a=1 / \sigma \nu z$.

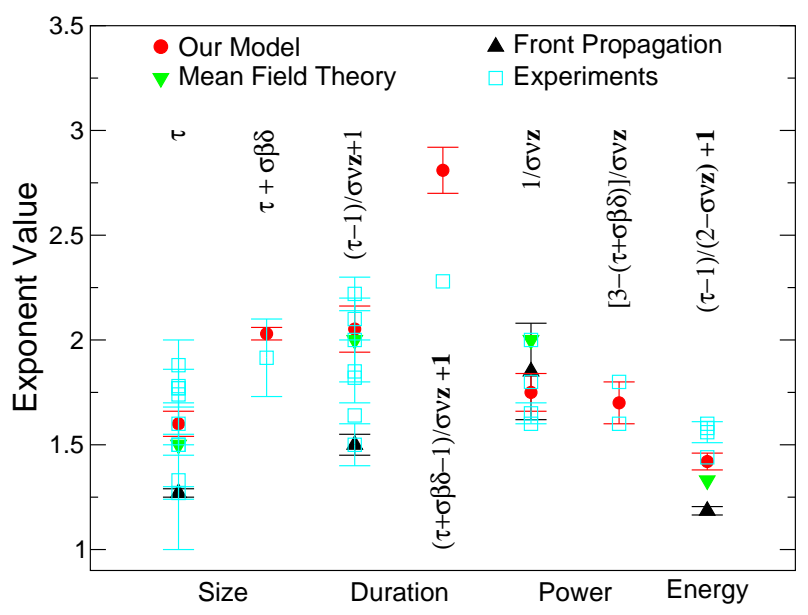

FIG. 11 Universal Critical Exponents vs. Experiment. (1) Different experiments on crackling noise in magnets measure different combinations of the universal critical exponents. Here we compare experimental measurements (see table I of reference (3)) to the theoretical predictions for three models: our model, the front-propagation model and the dipolar mean-field theory. Power laws giving the probability of getting an avalanche of a given size, duration, or energy at the critical point are shown; also shown is the critical exponent giving the power as a function of frequency (13) (due to the internal structure of the avalanches, figure 5). In each pair of columns, the first column includes only avalanches at external fields $H$ near $H_{c}$ where the largest avalanches occur, and the second column (when it exists) includes all avalanches. The various combinations of the basic critical exponents can be derived from exponent equality calculations similar to the one discussed in the text. Many of the experiments were done years before the theories were developed: many did not report error bars. All three theories do well (especially considering the possible systematic errors in fitting power laws to the experimental measurements: see in figure 3 how a bit away from $R_{c}$ the effective slopes change).(A more systematic and critical review of the exponent measurements in the literature may be found in Table I of reference (11). One may also find in section V.A of their paper a discussion of the relation between the experiments and the theoretical universality classes.)

There are several basic critical exponents, which arise in many different combinations depending on the physical property being studied. We've seen that at $R_{c}$ and near $H_{c}$ the probability of having an avalanche of size $S$ goes as $S^{-\tau} .{ }^{9}$ The cutoff in the avalanche size distribution in figure 3 gets larger as one approaches the critical

\footnotetext{
${ }^{9}$ We will see that when the entire hysteresis loop is considered this changes to $S^{-(\tau+\sigma \beta \delta)}$.
} 
disorder as $\left(R-R_{c}\right)^{-\sigma}$ (figure 3). The typical length of the largest avalanche goes as $\left(R-R_{c}\right)^{-\nu}$. The jump in the magnetization (figure 6) goes as $\left(R-R_{c}\right)^{\beta}$, and at $R_{c}$ the magnetization $\left(M-M_{c}\right) \sim\left(H-H_{c}\right)^{1 / \delta} .{ }^{10}$ Finally, we need to know how time rescales: the duration of an avalanche of spatial extent $L$ typically will go as $L^{z}$.

Any physical property that shows singular behavior at the critical point will have a critical exponent that can be written in terms of these basic ones (figure 11). ${ }^{11}$

To specialists in critical phenomena, these exponents are central; whole conversations will seem to rotate around various combinations of Greek letters. Critical exponents are one of the relatively easy things to calculate from the various analytic approaches, and so have attracted the most attention. They are derived from the eigenvalues of the linearized flows about the fixed point $\mathrm{S}^{*}$ in figure 7 Figure 12 shows our numerical estimates for several critical exponents in our model in various spatial dimensions, together with our $6-\epsilon$ expansions for them. Of course the key challenge is not to get analytical work to agree with numerics: it's to get theory to agree with experiment. Figure [1] shows that our model does rather well in describing a wide variety of experiments, but that the two rival models (with different flows around their fixed points) also fit.

Critical exponents are not the be-all and end-all: many other scaling predictions, explaining wide varieties of behavior, are quite easy to extract from numerical simulations. Universality extends even to those long length scale properties for which one cannot write formulas. Perhaps the most important of these other predictions are the universal scaling functions. For example, lets consider the time history of the avalanches, $V(t)$, denoting the number of domains flipping per unit time. (We call it $V$ because it's usually measured as a voltage in a pickup coil.) Each avalanche has large fluctuations, but one can average over many avalanches to get a typical shape. Figures 13 and 14 show averages over all avalanches of fixed duration $T$. Lets call this $\langle V\rangle(T, t)$. Universality again suggests that this average should be the same for experiment and a successful theory, apart from an overall shift in time and voltage scales:

$$
\langle V\rangle_{\exp }(T, t)=A\langle V\rangle_{\mathrm{th}}(T / B, t / B) .
$$

Comparing our model to itself with a shifted time scale becomes simple if we change variables: let $v(T, t / T)=$ $\langle V\rangle(T, t)$, so $v(T, t / T)=A v(T / B, t / T)$. Here $t / T$ is a particularly simple example of a scaling variable. Now, if we rescale time by a small factor $B=1 /(1-\delta)$, we have $v(T, t / T)=(1+b) v(t / T,(1-\delta) T)$. Again, making $\delta$ small we find $b v=T \partial v / \partial T$, with solution $v=v_{0} T^{b}$.

10 Don't confuse the small change in scale $\delta$ earlier with the critical exponent $\delta$ here.

${ }^{11}$ Indeed, there are relations even between these exponents: see section $\nabla]$

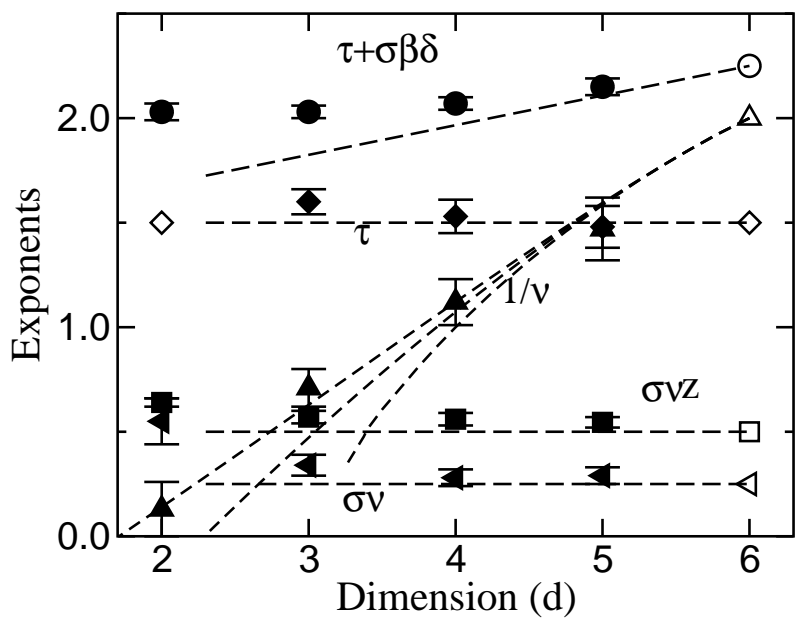

FIG. 12 Universal Critical Exponents in Various Spatial Dimensions. (1) We test our $\epsilon$-expansion predictions (27) by measuring the various critical exponents numerically in up to five spatial dimensions $(3 ; 28)$. The various exponents are described in the text. All of the exponents are calculated only to linear order in $\epsilon$, except for the correlation length exponent $\nu$, where we use results from other models. The agreement even in three dimensions is remarkably good, considering that we're expanding in $6-D$ where $D=3$ ! We should note that perturbing in dimension for our system is not only complicated, but also controversial. Our expansion uses the Martin Siggia Rose formalism $(29 ; 30 ; 31 ; 32 ; 33 ; 34 ; 35 ; 36)$ to describe a deterministic dynamical system without any temperature fluctuations, while the calculation for the equilibrium model involves temperature fluctuations and no history dependence at all. Even so, we have shown that our $6-\epsilon$ expansion for the critical exponents of the zero-temperature, nonequilibrium model maps to all orders in $\epsilon$ onto that of the equilibrium model. The $6-\epsilon$ expansion for the equilibrium model, however, has been controversial for decades (37). Recently the thermal $6-\epsilon$ expansion of the equilibrium model has been called into question $(37 ; 38 ; 39)$ not just due to non perturbative corrections, as was previously assumed, but due to previously neglected higher order terms in the expansion. The implications of this controversy for our non-equilibrium renormalization group treatments is not yet known.

However, the integration constant $v_{0}$ will now depend on $t / T, v 0=V(t / T)$, so we arrive at the scaling form

$$
\langle V\rangle(t, T)=T^{b} V(t / T),
$$

where the entire scaling function $V$ is a universal prediction of the theory.

Figures 13 and 14 show the universal scaling functions $V$ for two models and three experiments. For our model, we've drawn what are called scaling collapses, a simple but powerful way to both check that were in the scaling regime, and to measure the universal scaling function. Using the form of the scaling equation Eq.[2], we simply plot $T^{b}\langle V\rangle(t, T)$ versus $t / T$, for a series of long times $T$. All the plots fall onto the same curve. This tells us that our avalanches are large enough to be self-similar. (If in 


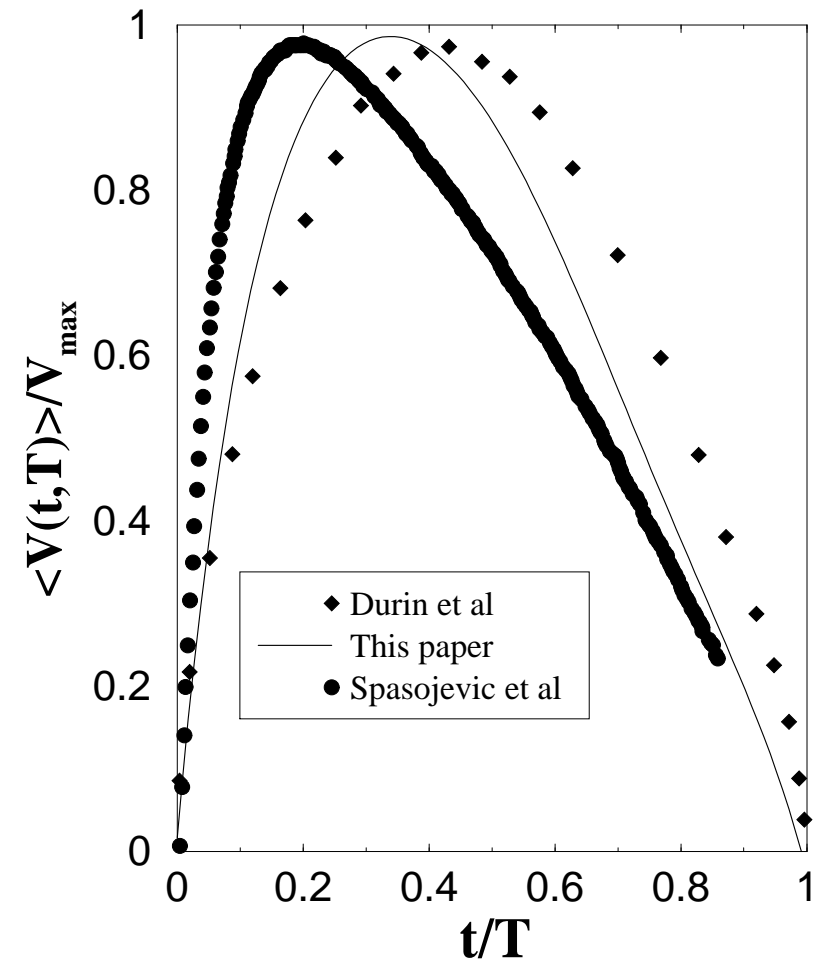

FIG. 13 Comparison of experimental average pulse shapes for fixed pulse duration, as measured by three different groups $(2 ; 11 ; 12 ; 40)$. Our theories don't only predict power laws: they should describe all behavior on long length and time scales (at least in a statistical sense). In particular, by fixing parameters one can predict what are called scaling functions. If we average the voltage as a function of time over all avalanches of a fixed duration, we get an average shape. In these three experiments, they find that this shape is the same for different durations: by rescaling the time by the duration $T$ and the voltage by the maximum of the average curve, the curves within a given experiment collapse onto one. The three experiments, however, do not all collapse onto the same curve. This could mean that they are in different universality classes, or that we don't understand this dynamical scaling completely.

your scaling collapse the corresponding plots do not all look alike, then any power laws you have measured are probably accidental.) It also provides us with a numerical evaluation of the scaling function $V$. Note that we use $1 / \sigma \nu z-1$ for the critical exponent $b$. This is an example of an exponent equality: easily derived from the fact that

$$
\langle S\rangle(T)=\int\langle V\rangle(t, T) d t=\int T^{b} V(t / T) d t \sim T^{b+1},
$$

and the scaling relation $\langle S\rangle(T) \sim T^{-1 / \sigma \nu z}$.

Notice that the two models and the three experiments have quite different shapes for $V$. How do we react to this? Our models are falsified if any of the predictions are shown to be wrong asymptotically on long length and time scales: hence our theory is either wrong (inapplicable to these systems) or somehow at least incom-

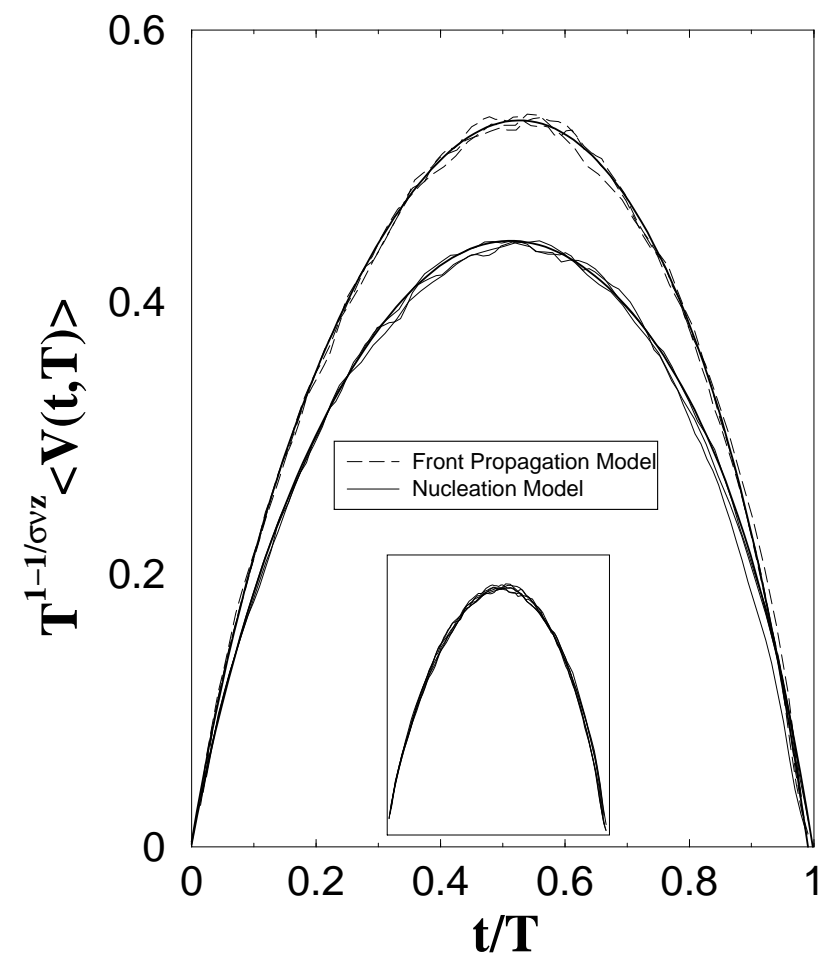

FIG. 14 Comparison of theoretical average pulse shape scaling functions for our nucleated model and the front propagation model (2). The front propagation models have $1 / \sigma \nu z=1.72 \pm 0.03$ in this collapse; our nucleation model has $1 / \sigma \nu z=1.75 \pm 0.03$ (in principle, there is no reason to believe these two should agree). The inset shows the two curves rescaled to the same height (the overall height is a nonuniversal feature): they are quantitatively different, but far more similar to one another than either is to the experimental curves in figure 13. The mean-field model apparently has a scaling function which is a perfect inverted parabola (41). The ABBM model, which is also mean-field like, interestingly has a different shape, that of one lobe of a sinusoid (42, eq. 102).

plete. Incorporating insights from careful experiments to refine the theoretical models has historically been crucial in the broad field of critical phenomena. The message we emphasize here is that scaling functions can provide a sharper tool for discriminating between different universality classes than critical exponents.

Broadly speaking, most common properties that involve large length and time scales have scaling forms: using self-similarity, one can write functions of $N$ variables in terms of scaling functions of $N-1$ variables: $F(x, y, z)=z^{-\alpha} F\left(x / z^{\beta}, y / z^{\gamma}\right)$. In the inset to figure 3] we show the scaling collapse for the integrated avalanche size distribution (derived as equation 10):

$$
D_{\text {int }}(S, R)=S^{-(\tau+\sigma \beta \delta)} \mathcal{D}_{\text {int }}\left(\left(R-R_{c}\right) / S^{-\sigma}\right) .
$$

This example illustrates that scaling works not only at $R_{c}$ but also near $R_{c}$; the unstable manifold in figure $\mathbf{7}$ governs the behavior for systems near the critical manifold 
C.

\section{MEASURING EXPONENTS AND SCALING FUNCTIONS}

The simulations provide a rich variety of measured quantities, each with a characteristic universal scaling form and associated critical exponents. In this section we focus on a few key ones of particular experimental significance. $^{12}$

We'll discuss the following properties obtained from the simulation

- the magnetization $M(H, R)$ as a function of the external field $H$.

- the avalanche size distribution integrated over the field $H, D_{\text {int }}(S, R)$.

- the avalanche correlation function integrated over the field $H, G_{\text {int }}(x, R)$.

- the distribution of avalanche durations $D_{t}^{(i n t)}(S, t)$ as a function of the avalanche size $S$, at $R=R_{c}$, integrated over the field $H$.

- the energy spectrum $E(\omega)$ of the Barkhausen noise for various models at criticality.

\section{A. Magnetization Curves}

Unfortunately the most obvious measured quantity in our simulations, the magnetization curve $M(H)$, is the one which collapses least well in our simulations. We start with it nonetheless.

The top figure 15] shows the magnetization curves obtained from our simulation in 3 dimensions for several values of the disorder $R$. As the disorder $R$ is decreased, a discontinuity or jump in the magnetization curve appears where a single avalanche occupies a large fraction of the total system. In the thermodynamic limit this would be the infinite avalanche: the largest disorder at which it occurs is the critical disorder $R_{c}$. For finite size systems, like the ones we use in our simulation, we observe an avalanche which spans the system at a higher disorder, which gradually approaches $R_{c}$ as the system size grows.

Figure [16] shows the slope $d M / d H$ and its scaling collapse. By using this derivative, the critical region is emphasized as the peak in the curve, and the dependence on the parameter $M_{c}$ drops out. The lower graphs in figure [15] and 16] show the scaling collapses of the magnetization and its slope. Clearly in neither case is all the data collapsing onto a single curve. This would be distressing, were it not for the fact that this also occurs in

12 This section follows closely the presentation in (28).
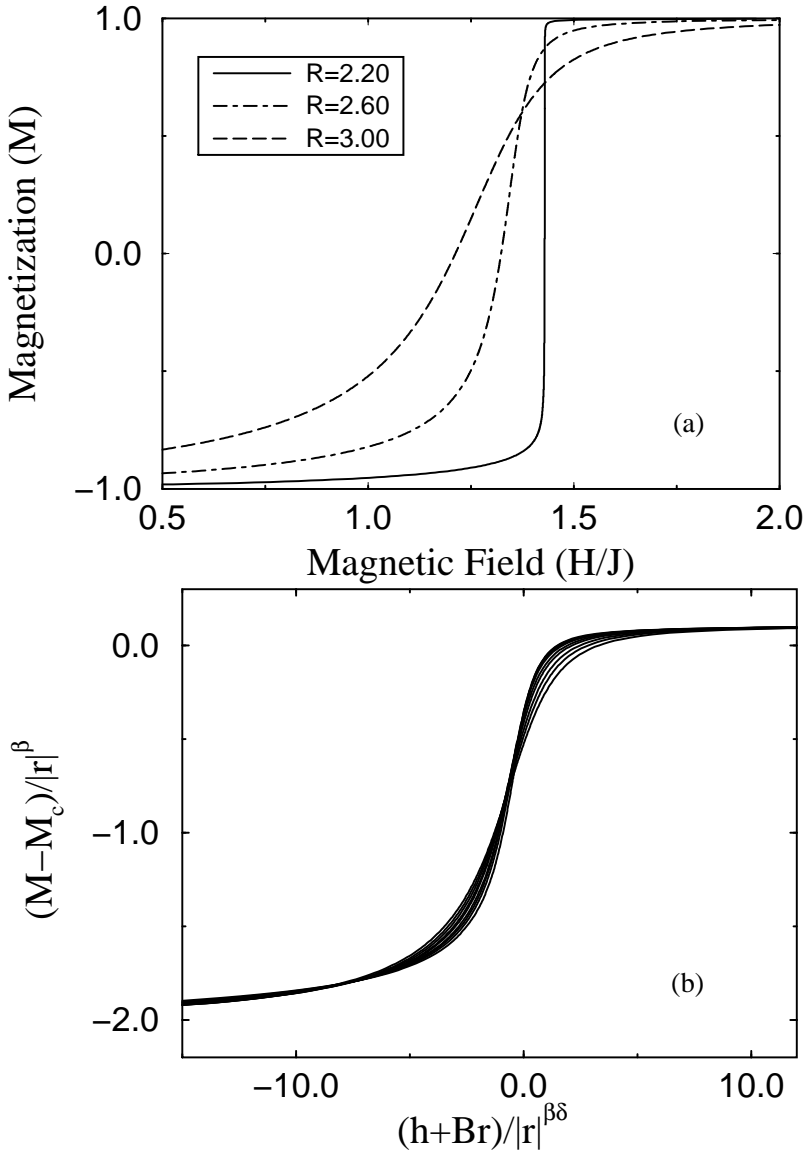

FIG. $15 M(H)$ curves at different disorders, and (poor) universal scaling collapse. (28)

mean field theory (43) at a similar distance to the critical point.

Because the scaling of the magnetization is so bad, we use other quantities to estimate the critical exponents and the location of the critical point (Tables I and III). Fixing these quantities, we use the collapse of the $d M / d H$ curves to extract the rotation $B$ mixing the experimental variables $r$ and $h$ into the scaling variable $h^{\prime}=h+B r$ (equation \$13 and following discussion). Recent work suggests that much of the difficulty in collapsing the magnetization curves is surmountable by adding analytical corrections to scaling (44).

\section{B. Avalanche Size Distribution}

In our model the spins flip in avalanches: each spin can kick over one or more neighbors in a cascade. These avalanches come in different sizes. The integrated avalanche size distribution is the size distribution of all the avalanches that occur in one branch of the hysteresis loop (for $H$ from $-\infty$ to $\infty$ ). Figure 3 (3) shows some of the raw data (thick lines) in 3 dimensions. Note that the curves follow an approximate power law behav- 

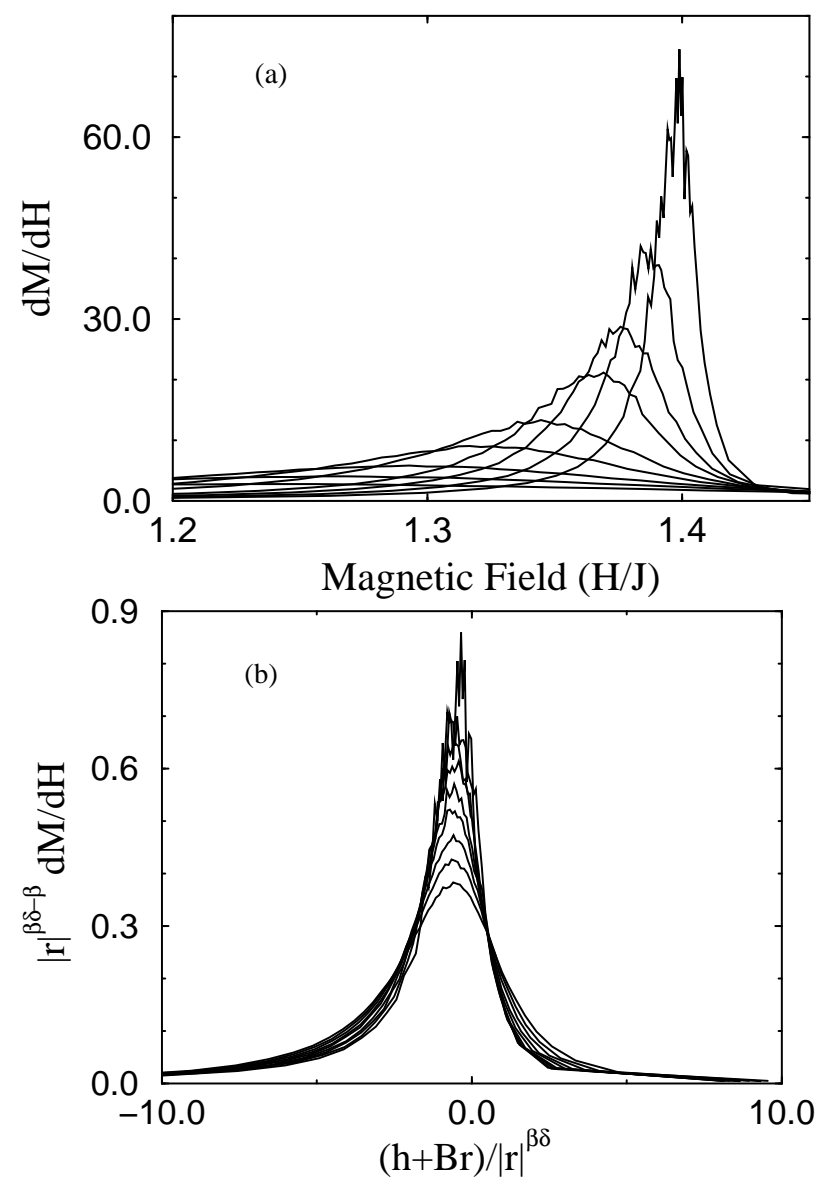

FIG. $16 \frac{d M}{d H}(H)$ curves at different disorders, and (poor) universal scaling collapse. (28) While the curves are not collapsing onto a single curve, the quality of the collapse is quite similar to that found at similar distances from $R_{c}$ in mean field theory (43), for which we know analytically that scaling works as $R \rightarrow R_{c}$.

ior over several decades. Even $50 \%$ away from criticality (at $R=3.2$ ), there are still two decades of scaling, which implies that the critical region is large. In experiments, a few decades of scaling could be interpreted in terms of self-organized criticality. However, our model and simulation suggest that several decades of power law scaling can still be present rather far from the critical point (note that the size of the critical region is non-universal). The slope of the $\log -\log$ avalanche size distribution at $R_{c}$ gives the critical exponent $\tau+\sigma \beta \delta$. Notice, however, that the apparent slopes in figure 3 continue to change even after several decades of apparent scaling is obtained. The cutoff in the power law diverges as the critical disorder $R_{c}$ is approached. This cutoff size scales as $S \sim|r|^{-1 / \sigma}$.

These critical exponents can be obtained by using a scaling collapse for the curves of figure 3 shown in the inset. The scaling form for the avalanche sizes as a function of $R$ and $H$ is

$$
D(S, R, H) \sim S^{-\tau} \mathcal{D}\left(S^{\sigma}|r|, h / r^{\beta \delta}\right) .
$$

We can find the scaling form for the distribution of avalanche sizes integrated over $H$ by integrating this formula, changing variables to $y=h / r^{\beta \delta}$, and rewriting the resulting formula in terms of $S$ to a power times a function of $S^{\sigma} r$. For $R>R_{c}$,

$$
\begin{aligned}
D_{\text {int }}(S, R) & =\int D(S, R, H) d H \\
& \propto \int S^{-\tau} \mathcal{D}\left(S^{\sigma} r, h / r^{\beta \delta}\right) d h \\
& =S^{-\tau} r^{\beta \delta} \int \mathcal{D}\left(S^{\sigma} r, y\right) d y \\
& =S^{-(\tau+\sigma \beta \delta)}\left(S^{\sigma} r\right)^{\beta \delta} \int \mathcal{D}\left(S^{\sigma} r, y\right) d y \\
& =S^{-(\tau+\sigma \beta \delta)} \mathcal{D}_{+}^{(i n t)}\left(S^{\sigma} r\right)
\end{aligned}
$$

where $\mathcal{D}_{+}^{(i n t)}$ is the scaling function for the integrated avalanche size distribution (the + sign indicates that the collapsed curves are for $R>R_{c}$ ). We are sufficiently far from the critical point that corrections to scaling are important: as described in reference (43), we do collapses for small ranges of $R$ and then linearly extrapolate the best-fit critical exponents to $R_{c}$. We estimate from this curve that the critical exponents $\tau+\sigma \beta \delta=2.03$ and $\sigma=0.24$

The scaling function $\mathcal{D}_{+}^{(i n t)}(X)$ with $X=S^{\sigma}|r|$ is a universal prediction of our model. To facilitate comparisons with experiments, we fit a curve to the data collapse in the inset of figure 3. We have fit the scaling collapses in dimensions 3,4 , and 5 to a phenomenological form of an exponential times a polynomial. In three dimensions, our fit is

$$
\begin{array}{r}
\mathcal{D}_{+}^{(i n t)}(X)=e^{-0.789 X^{1 / \sigma}} \times \\
\left(0.021+0.002 X+0.531 X^{2}-0.266 X^{3}+0.261 X^{4}\right)
\end{array}
$$

where $1 / \sigma=4.20$. The distribution curves obtained using the above fit are plotted (thin lines in figure 3) alongside the raw data (thick lines). They agree remarkably well even far above $R_{c}$. We should recall though, that the fitted curve to the collapsed data can differ from the "real" scaling function even for large sizes and close to the critical disorder (in mean field (43) the error in the corresponding curve was about $10 \%$ ). The scaling function in the inset of figure 3 has a peculiar shape: it grows by a factor of ten before cutting off. The consequence of this bump in the shape is that in the simulations it takes many decades in the size distribution for the slope to converge to the asymptotic power law. This can be seen from the comparison between a straight line fit through the $R=2.25$ (billion spin) simulation in figure 3 and the asymptotic power law $S^{-2.03}$ obtained from extrapolating the scaling collapses (thick dashed straight line in the same figure). A similar bump exists in other dimensions and mean field as well. Figure 17 shows the scaling functions in different dimensions and in mean field. In this graph, the scaling functions are normalized to one and the peaks are aligned (the scaling forms allow this). The 
curves plotted in figure 17 are not raw data but fits to the scaling collapse in each dimensions, as was done in the inset of figure 3

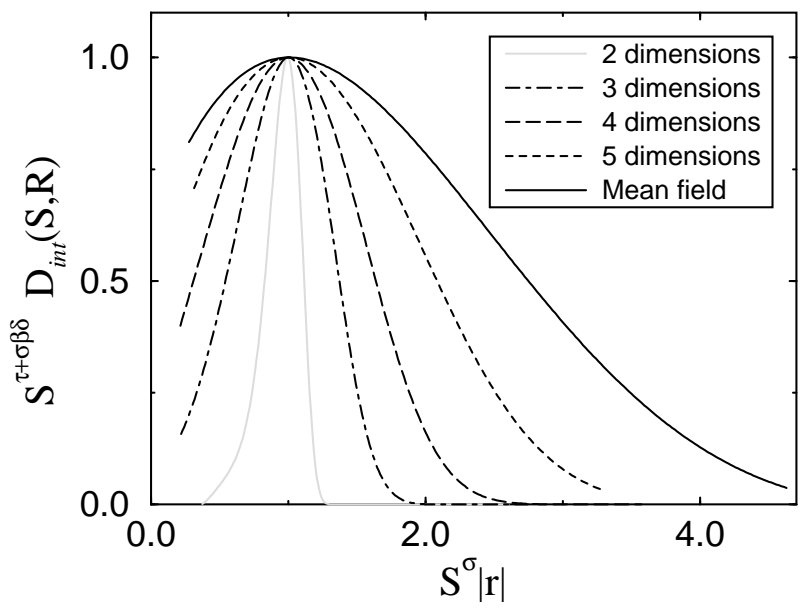

FIG. 17 Universal scaling functions $\mathcal{D}_{\text {int }}\left(S^{\sigma}|r|\right)$ for the avalanche size distributions in different spatial dimensions. (28) The inset of figure 3 shows the scaling collapse for three dimensions.

It is clear from the figure that the growing bump in the scaling curves as the dimension decreases is a foreshadowing of a zero in the scaling curve in two dimensions.

\section{Avalanche Correlations}

The avalanche correlation function $G(x, R, H)$ measures the probability that the initial spin of an avalanche will trigger, in that avalanche, another spin a distance $x$ away. From the renormalization group description (27; 45), close to the critical point and for large distances $x$, the correlation function is given by:

$$
G(x, R, H) \sim \frac{1}{x^{d-2+\eta}} \mathcal{G}_{ \pm}(x / \xi(r, h))
$$

where $r$ and $h$ are respectively the reduced disorder and field, $\mathcal{G}_{ \pm}$( \pm indicates the sign of $r$ ) is the scaling function, $d$ is the dimension, $\xi$ is the correlation length, and $\eta$ is called the "anomalous dimension". Corrections can be shown to be subdominant (appendix $\mathrm{A}$ ). The correlation length $\xi(r, h)$ is a macroscopic length scale in the system which is on the order of the mean linear extent of the largest avalanches. At the critical field $H_{c}(\mathrm{~h}=0)$ and near $R_{c}$, the correlation length scales like $\xi \sim|r|^{-\nu}$, while for small field $h$ it is given by

$$
\xi \sim|r|^{-\nu} \mathcal{Y}_{ \pm}\left(h /|r|^{\beta \delta}\right)
$$

where $\mathcal{Y}_{ \pm}$is a universal scaling function. The avalanche correlation function should not be confused with the cluster or "spin-spin" correlation which measures the probability that two spins a distance $x$ away have the same
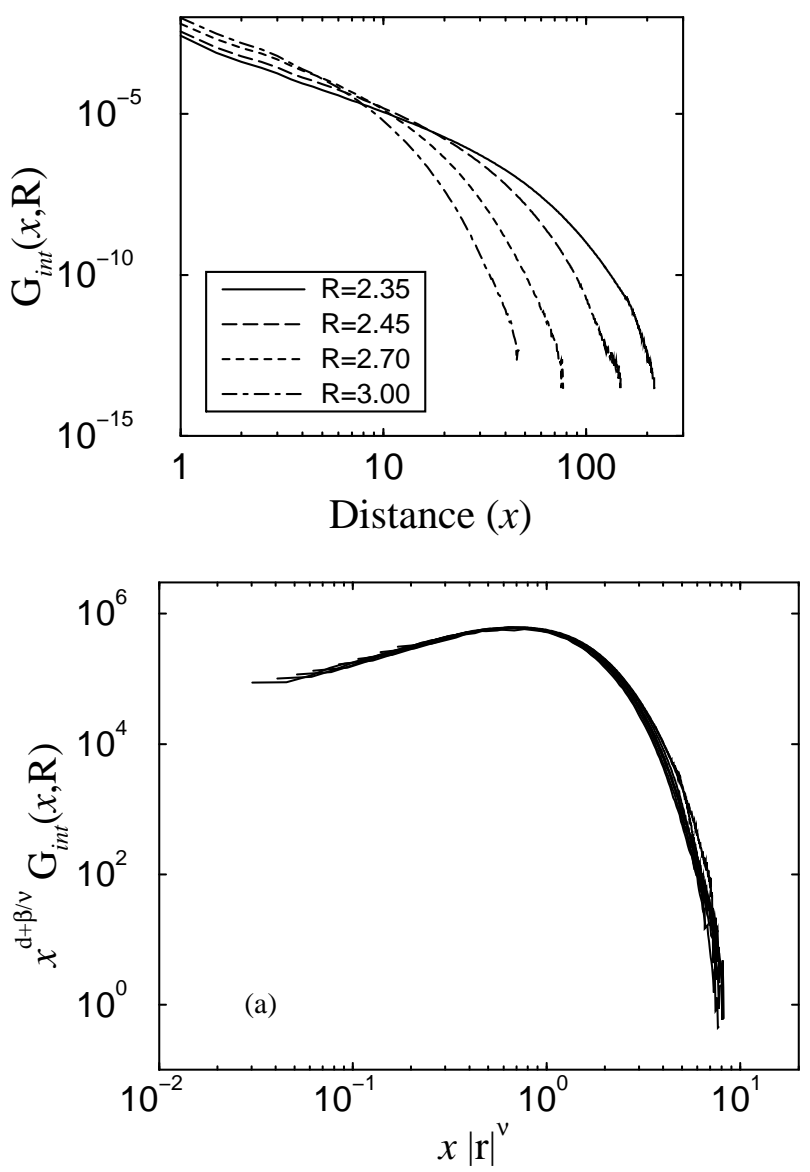

FIG. 18 Avalanche correlation function integrated over field $H$ at various disorders, with scaling collapse (28).

value. (The algebraic decay for this other, spin-spin correlation function at the critical point $(r=0$ and $h=0)$, is $1 / x^{d-4+\tilde{\eta}}$ (45).)

We've mostly used, for historical reasons, a slightly different avalanche correlation function, which scales the same way as the "triggered" correlation function $G$ described above. Our function basically ignores the difference between the triggering spin and the other spins in the avalanche: alternatively, it calculates for avalanches of size $S$ the correlation function for pairs of spins, and then averages over all avalanches (weighting each avalanche equally). We've checked that our correlation function agrees to within $3 \%$ with the "triggered" correlation function described above, for $R>R_{c}$ in three dimensions and above. (In two dimensions, the two definitions differ more substantially, but appear to scale in the same way.)

We have measured the avalanche correlation function integrated over the field $H$, for $R>R_{c}$. For every avalanche that occurs between $H=-\infty$ and $H=+\infty$, we keep a count on the number of times a distance $x$ occurs in the avalanche. The spanning avalanches are 
not included in our correlation measurement. Figure 18 shows several avalanche correlation curves in 3 dimensions for $L=320$. The scaling form for the avalanche correlation function integrated over the field $H$, close to the critical point and for large distances $x$, is obtained by integrating equation (12):

$$
G_{\text {int }}(x, R) \sim \int \frac{1}{x^{d-2+\eta}} \mathcal{G}_{ \pm}(x / \xi(r, h)) d h
$$

Using equation (13) and defining $u=h /|r|^{\beta \delta}$, equation (14) becomes:

$$
G_{i n t}(x, R) \sim|r|^{\beta \delta} x^{-(d-2+\eta)} \int \mathcal{G}_{ \pm}\left(x /|r|^{-\nu} \mathcal{Y}_{ \pm}(u)\right) d u
$$

The integral $(\mathcal{I})$ in equation (15) is a function of $x|r|^{\nu}$ and can be written as:

$$
\mathcal{I}=\left(x|r|^{\nu}\right)^{-\beta \delta / \nu} \widetilde{\mathcal{G}}_{ \pm}\left(x|r|^{\nu}\right)
$$

to obtain the scaling form:

$$
G_{i n t}(x, R) \sim \frac{1}{x^{d+\beta / \nu}} \widetilde{\mathcal{G}}_{ \pm}\left(x|r|^{\nu}\right)
$$

where we have used the scaling relation $(2-\eta) \nu=\beta \delta-\beta$ (see (45) for the derivation).

The bottom figure 18 shows the integrated avalanche correlation curves collapse in 3 dimensions for $L=320$ and $R>R_{c}$. The exponent $\nu$ is obtained from such collapses by extrapolating to $R=R_{c}$ as was done for other collapses (43). The exponent $\beta / \nu$ can be obtained from these collapses too, but we found it better to use the jump in magnetization (28), which near the critical point involves several spanning avalanches. Recent work $(46 ; 47$; 48) suggests that the jump in magnetization may be dominated by only one of these spanning avalanches, and their work suggests that there may be two exponents related to our $\beta$, one substantially larger (their $\beta_{c} \sim 0.15 \pm 0.08$ ). The value of $\beta / \nu$ listed in Table I is derived exclusively from the magnetization discontinuity collapses.

One of the most artificial features of our model is that the domains are arranged in a regular grid. It is thus important to check that this grid is not important for our scaling properties: if the avalanches looked noticeably cubical, we would have problems. We have looked for possible anisotropies in the integrated avalanche correlation function in 2 and 3 dimensions. The anisotropic integrated avalanche correlation functions are measured along "generalized diagonals": one along the three axis, the second along the six face diagonals, and the third along the four body diagonals. We compare the integrated avalanche correlation function and the anisotropic integrated avalanche correlation functions to each other, and find no anisotropies in the correlation, as can be seen from figure 19 Spherical symmetry emerges at the critical point on long length and time scales.

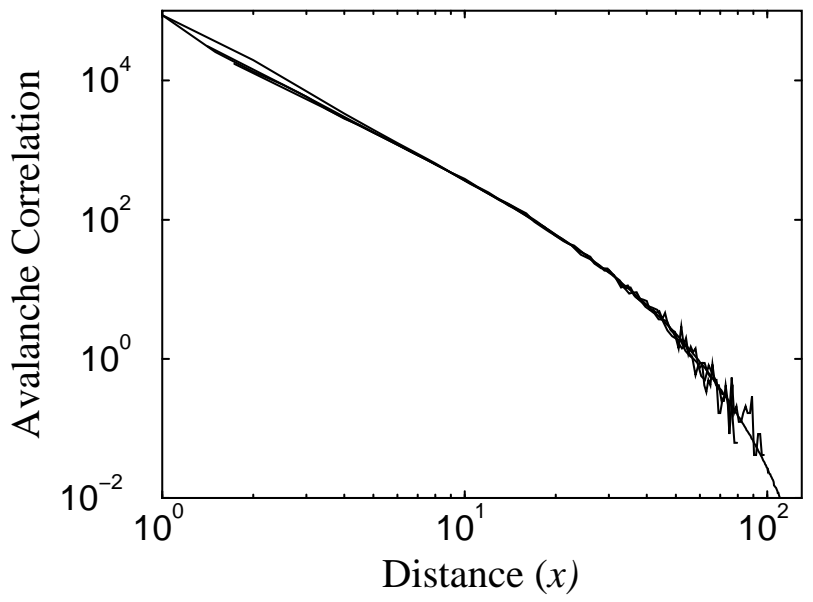

FIG. 19 Anisotropies in the avalanche correlation function. Four curves are shown on the graph: one is the avalanche correlation function integrated over the field $H$ (as in figure 18), while the other three are measurements of the correlation along the three axis, the six face diagonals, and the four body diagonals. Avalanches involving more than four spins show no noticeable anisotropy: the critical point appears to have spherical symmetry. The same result is found in 2 dimensions.

\section{Avalanche Duration Measurement}

The scaling relation between the duration $T$ of an avalanche (the time it takes to occur) and the linear size $\xi$ of the avalanche defines the critical exponent $z$

$$
T \sim \xi^{z}
$$

The exponent $z$ is known as the dynamical critical exponent. Equation (18) gives the scaling for the time it takes for a spin to "feel" the effect of another a distance $\xi$ away. Since the correlation length $\xi$ scales like $r^{-\nu}$ close to the critical disorder, and the characteristic size $S$ as $r^{-1 / \sigma}$, the duration $T$ then scales with avalanche size as:

$$
T \sim S^{\sigma \nu z}
$$

In our simulation, we measure the distribution of durations for each avalanche size $S$. The probability $D_{t}(S, R, H, T)$ that an avalanche of size $S$ will be of duration $T$ close to the critical field $H_{c}$ and critical disorder $R_{c}$ has the scaling form

$$
D_{t}(S, R, H, T) \sim S^{-q} \mathcal{D}_{ \pm}^{(t)}\left(S^{\sigma}|r|, h /|r|^{\beta \delta}, T / S^{\sigma \nu z}\right)
$$

where $q=\tau+\sigma \nu z$, and is defined such that

$$
\begin{array}{r}
\int_{-\infty}^{+\infty} \int_{1}^{\infty} D_{t}(S, R, H, T) d H d t= \\
S^{-(\tau+\sigma \beta \delta)} \mathcal{D}_{ \pm}^{(\text {int })}\left(S^{\sigma}|r|\right)
\end{array}
$$

where $\mathcal{D}_{ \pm}^{(\text {int })}$ was defined in the integrated avalanche size distribution section. The avalanche time distribution integrated over the field $H$, at the critical disorder $(r=0)$ 
is:

$$
D_{t}^{(i n t)}(S, T) \sim t^{-(\tau+\sigma \beta \delta+\sigma \nu z) / \sigma \nu z} \mathcal{D}_{t}^{(i n t)}\left(T / S^{\sigma \nu z}\right)
$$

as obtained from equation (20) (reference (43)).
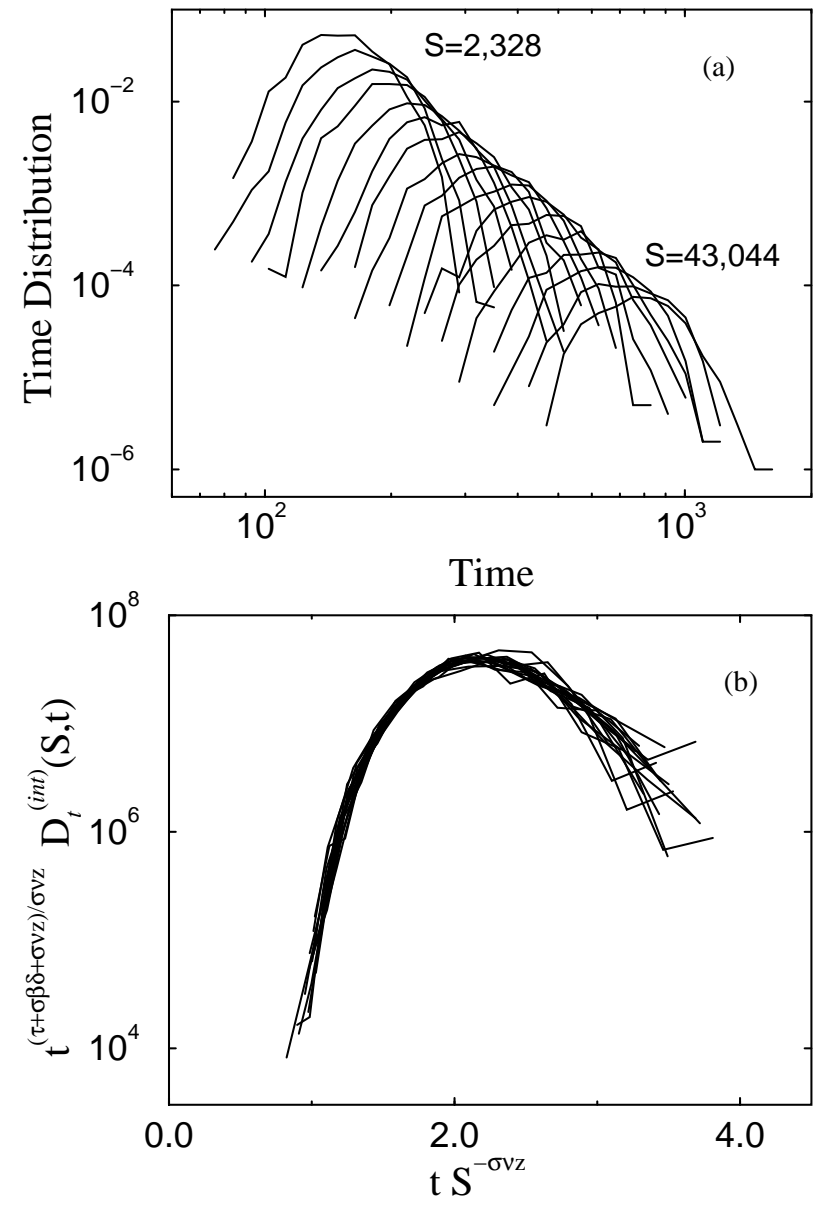

FIG. 20 Distribution of avalanche durations for avalanches of fixed size $S$, for various sizes $S$, and their scaling collapse. (28)

Figures 20] show the avalanche time distribution integrated over the field $H$ for different avalanche sizes, and a collapse of these curves using the above scaling form.

\section{E. Energy Spectrum}

The energy spectrum of the voltage $V(t)$ as a function of time for our model (given by adding up the the voltage traces of each avalanche, as in figure 5),

$$
E(\omega)=\int e^{-i \omega \tau}\langle V(t) V(t+\tau)\rangle=|\widetilde{V}(\omega)|^{2}
$$

is a commonly measured experimental quantity. ${ }^{13}$ There are two distinct contributions to this power spectrum:

\footnotetext{
13 The power spectrum is $E(\omega)$ divided by the integration time.
}
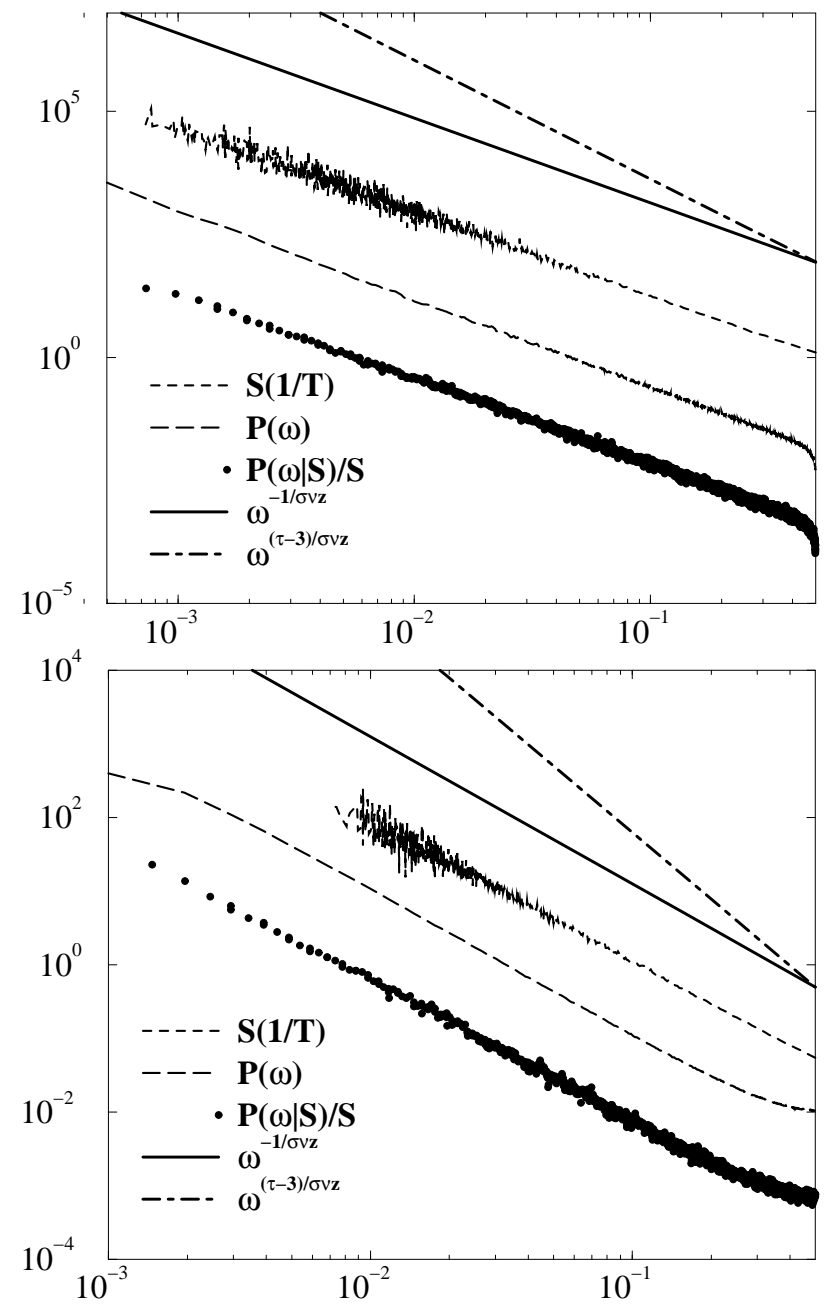

FIG. 21 Power spectrum scaling and relatives. Top: short-range model; bottom: mean field (long-range dipolar forces (13).)

(1) at high frequencies, the incoherent sum of the power spectra $E_{\text {inc }}$ of the individual avalanches, and (2) at low frequencies, a term representing the correlations between avalanches. As the system is forced more and more slowly, the internal dynamics of the avalanches is unchanged but their separation in time increases, so these two contributions separate. Typical experiments seem to be dominated by the contribution (1) from the internal dynamics within an avalanche (13). In this section, the results we discuss are valid for all three models of hysteresis. ${ }^{14}$ See also (42) in this volume for a more complete discussion of our power spectrum theory and the experimental context.

14 In the short-range model, we concentrate on the case where we measure only near $R_{c}$ and $H_{c}$ : we do not integrate over the loop. Integrating over the loop yields $\tau$ very close to two, where both forms of the scaling should compete. 
There have been a number of naive arguments for the power law for $E(\omega)$, stretching back to 1972 (3; 27; 40; 49), which yield a power law which is only valid if $\tau>2$ :

$$
E_{\text {naive }}(\omega) \propto \omega^{-(3-\tau) / \sigma \nu z} \quad \text { (only for } \tau>2 \text { ). }
$$

Let $S_{\max }$ be the typical largest avalanche in the system, say because the system is finite in size. At root, these derivations fail for $\tau<2$ because

$$
\int_{0}^{S_{\max }} S \mathcal{D}(S) d S=\int_{0}^{S_{\max }} S^{1-\tau} d S=S_{\max }^{2-\tau}
$$

diverges. Since there are $S$ spins in each avalanche, it would seem that equation 25] should represent the number of spins in the system. This is roughly correct for $\tau>2$, but for $\tau<2$ the amplitude of the power law $D(S)=$ $D_{0} S^{-\tau}$ depends on this cutoff $D_{0} \sim S_{\max }^{-2}$. Continuing a detailed analysis (13), we get a different scaling form

$$
E_{\text {naive }}(\omega) \propto \omega^{-1 / \sigma \nu z} \quad(\text { for } \tau<2) .
$$

It is not a surprise that this new prediction fixes a number of discrepancies between theory and experiments (13).

Figure 21 shows the power spectrum $P(\omega)$ (equal to $E(\omega)$ divided by the duration of the measurement), together with four other curves, for both the frontpropagation model and the infinite--range model (13). The top two curves are the naive power law (equation 24 obviously not correct) and the correct law (equation [26). We compare with the relation between the avalanche size $S$ and the duration $T$ (equation [19), which has the same combination of critical exponents $S \sim(1 / T)^{-1 / \sigma \nu z}$. We also show that the energy spectrum of individual large avalanches is proportional to $S$ and has the same power law in frequency $\omega$ as that of the entire time series, so we plot $P(\omega \mid S) / S$ for a variety of avalanche sizes $S$.

\section{F. Tables of Results}

Here we summarize, from (28), our numerical estimates of the universal critical exponents and various non-universal quantities used in the scaling collapses above. ${ }^{15}$

\begin{tabular}{ccccc} 
measured exponents & $3 \mathrm{~d}$ & $4 \mathrm{~d}$ & $5 \mathrm{~d}$ & mean field \\
\hline $1 / \nu$ & $0.71 \pm 0.09$ & $1.12 \pm 0.11$ & $1.47 \pm 0.15$ & 2 \\
$\theta$ & $0.015 \pm 0.015$ & $0.32 \pm 0.06$ & $1.03 \pm 0.10$ & 1 \\
$(\tau+\sigma \beta \delta-3) / \sigma \nu$ & $-2.90 \pm 0.16$ & $-3.20 \pm 0.24$ & $-2.95 \pm 0.13$ & -3 \\
$1 / \sigma$ & $4.2 \pm 0.3$ & $3.20 \pm 0.25$ & $2.35 \pm 0.25$ & 2 \\
$\tau+\sigma \beta \delta$ & $2.03 \pm 0.03$ & $2.07 \pm 0.03$ & $2.15 \pm 0.04$ & $9 / 4$ \\
$\tau$ & $1.60 \pm 0.06$ & $1.53 \pm 0.08$ & $1.48 \pm 0.10$ & $3 / 2$ \\
$d+\beta / \nu$ & $3.07 \pm 0.30$ & $4.15 \pm 0.20$ & $5.1 \pm 0.4$ & $7\left(\right.$ at $\left.d_{c}=6\right)$ \\
$\beta / \nu$ & $0.025 \pm 0.020$ & $0.19 \pm 0.05$ & $0.37 \pm 0.08$ & 1 \\
$\sigma \nu z$ & $0.57 \pm 0.03$ & $0.56 \pm 0.03$ & $0.545 \pm 0.025$ & $1 / 2$
\end{tabular}

Table I. Measured universal critical exponents. Values for the exponents extracted from scaling collapses in 3, 4, and 5 dimensions. The mean field values are calculated analytically $(23 ; 45) . \nu$ is the correlation length exponent and is found from collapses of avalanche correlations, number of spanning avalanches, and moments of the avalanche size distribution data. The exponent $\theta$ is a measure of the number of spanning avalanches and is obtained from collapses of that data. $(\tau+\sigma \beta \delta-3) / \sigma \nu$ is obtained from the second moments of the avalanche size distribution collapses. $1 / \sigma$ is associated with the cutoff in the power law distribution of avalanche sizes integrated over the field $H$, while $\tau+\sigma \beta \delta$ gives the slope of that distribution. $\tau$ is obtained from the binned avalanche size distribution collapses (28). $d+\beta / \nu$ is obtained from avalanche correlation collapses and $\beta / \nu$ from magnetization discontinuity collapses. $\sigma \nu z$ is the exponent combination for the time distribution of avalanche sizes and is extracted from that data. Error bars are based on variations in the results based on different approaches to the analysis: statistical fluctuations are typically smaller.

\footnotetext{
15 Some of the scaling collapses shown used exponents slightly different from those in the tables: see (28).
} 


\begin{tabular}{ccccc} 
calculated exponents & $3 \mathrm{~d}$ & $4 \mathrm{~d}$ & $5 \mathrm{~d}$ & mean field \\
\hline$\sigma \beta \delta$ & $0.43 \pm 0.07$ & $0.54 \pm 0.08$ & $0.67 \pm 0.11$ & $3 / 4$ \\
$\beta \delta$ & $1.81 \pm 0.32$ & $1.73 \pm 0.29$ & $1.57 \pm 0.31$ & $3 / 2$ \\
$\beta$ & $0.035 \pm 0.028$ & $0.169 \pm 0.048$ & $0.252 \pm 0.060$ & $1 / 2$ \\
$\sigma \nu$ & $0.34 \pm 0.05$ & $0.28 \pm 0.04$ & $0.29 \pm 0.04$ & $1 / 4$ \\
$\eta=2+(\beta-\beta \delta) / \nu$ & $0.73 \pm 0.28$ & $0.25 \pm 0.38$ & $0.06 \pm 0.51$ & 0
\end{tabular}

Table II. Calculated universal critical exponents. Values for exponents in 3 , 4 , and 5 dimensions that are not extracted directly from scaling collapses, but instead are derived from Table I and the exponent relations (see section VI). The mean field values are obtained analytically (23; 45). Both $\sigma \beta \delta$ and $\beta \delta$ could have larger systematic errors than the errors listed here (28). Recent work (46) on small systems, but with sophisticated scaling analysis, suggests a larger value for $\beta$.

\begin{tabular}{ccccc} 
& $3 \mathrm{~d}$ & $4 \mathrm{~d}$ & $5 \mathrm{~d}$ & mean field \\
\hline$R_{c}$ & $2.16 \pm 0.03$ & $4.10 \pm 0.02$ & $5.96 \pm 0.02$ & 0.79788456 \\
$H_{c}$ & $1.435 \pm 0.004$ & $1.265 \pm 0.007$ & $1.175 \pm 0.004$ & 0 \\
$B$ & $0.39 \pm 0.08$ & $0.46 \pm 0.05$ & $0.23 \pm 0.08$ & 0
\end{tabular}

Table III. Non-universal scaling variables. Numerical values for the critical disorders and fields, and the rotation parameter $B$ (equation $\$ 13$ and subsequent discussion), in 3, 4, and 5 dimensions extracted from scaling collapses. The critical disorder is obtained from collapses of the spanning avalanches and the second moments of the avalanche size distribution. The critical field is obtained from the binned avalanche size distribution (28) and the magnetization curves. $H_{c}$ is affected by finite sizes, and systematic errors could be larger than the ones listed here. The mean field values are calculated analytically (23; 45). The rotation $B$ is obtained from the $d M / d H$ collapses.

\section{EXPONENT RELATIONS}

In the following sections we list various exponent relations for the nonequilibrium RFIM, for which we give detailed arguments in $(27 ; 50)$.

\section{A. Exponent equalities}

The exponents introduced above are related by the following exponent equalities:

$$
\begin{gathered}
\beta-\beta \delta=(\tau-2) / \sigma \text { if } \tau<2, \\
(2-\eta) \nu=\beta \delta-\beta, \\
\beta=\frac{\nu}{2}(d-4+\bar{\eta}),
\end{gathered}
$$

and

$$
\delta=(d-2 \eta+\bar{\eta}) /(d-4+\bar{\eta}) .
$$

(The latter three equations are not independent and are also valid in the equilibrium random-field Ising model).

\section{B. Two violations of hyperscaling}

In the nonequilibrium RFIM there are two different violations of hyperscaling.

(1) In (27; 50), we show that the connectivity hyperscaling relation $1 / \sigma=d \nu-\beta$ from percolation is violated in our system. There is a new exponent $\theta$ defined by

$$
1 / \sigma=(d-\theta) \nu-\beta
$$

with $\theta \nu=1 / 2-\epsilon / 6+0\left(\epsilon^{2}\right)$ and $\theta \nu=0.021 \pm 0.021$ in three dimensions. $\theta$ is related to the number of system spanning avalanches observed during a sweep through the hysteresis loop: see also (46).

(2) As we discuss in (27) there is a mapping of the perturbation theory for our problem to that of the equilibrium random-field Ising model to all orders in $\epsilon$. From that mapping we deduce the breakdown of an infamous ("energy")-hyperscaling relation

$$
\beta+\beta \delta=(d-\bar{\theta}) \nu,
$$

with a new exponent $\bar{\theta}$, which has caused much controversy in the case of the equilibrium random-field Ising model.

In $(27 ; 50)$ we discuss the relation of the exponent $\bar{\theta}$ to the energy output of the avalanches. The $\epsilon$ expansion yields $\bar{\theta}=2$ to all orders in $\epsilon$. Nonperturbative corrections are expected to lead to deviations of $\bar{\theta}$ from 2 as the dimension is lowered. The same is true in the case of the equilibrium RFIM. The numerical result in three 
dimensions is $\bar{\theta}=1.69 \pm 0.28$. (In the three-dimensional equilibrium RFIM (51) it is $\left.\bar{\theta}_{e q}=1.5 \pm 0.4\right)$.

Another strictly perturbative exponent equality, which is also obtained from the perturbative mapping to the random-field Ising model is given by

$$
\bar{\eta}=\eta
$$

It, too, is expected to be violated by nonperturbative corrections below six dimensions.

\section{Exponent inequalities}

In $(27 ; 50)$ we give arguments for the following two exponent-inequalities. ${ }^{16}$

$$
\nu / \beta \delta \geq 2 / d
$$

which is formally equivalent to the "Schwartz-Soffer" inequality, $\bar{\nu} \leq 2 \nu$, first derived for the equilibrium random-field Ising model, and

$$
\nu \geq 2 / d,
$$

which is a weaker bound than Eq. (3) so long as $\beta \delta \geq 1$, as appears to be the case both theoretically and numerically at least for $d \geq 3$.

\section{FINITE SWEEPRATE}

Originally the nonequilibrium RFIM was studied in the adiabatic limit, where the external field is kept constant during each spin flip avalanche and only increased between subsequent avalanches. In real experiments, however, the driving field $H$ is typically increased at a finite rate $\Omega$ such that $H=\Omega t$ where $t$ is time. This finite rate allows for new avalanches to be triggered by the increasing external field while an earlier avalanche is still propagating. In $(52 ; 53)$ the effect of finite field sweep rates on the power spectra and avalanche size distributions is discussed in detail for a large class of systems with crackling noise. In particular, it is asked how the scaling behavior of the avalanche size and duration distribution and the power spectra of Barkhausen noise or crackling noise in general depend on the field sweep rate $\Omega$. One of the results is an exponent inequality as a criteria for the relevance of adding a small driving rate $\Omega>0$ to the adiabatic case $\Omega \rightarrow 0$ : If in the adiabatic case the avalanche duration distribution scales as $D(T) \sim T^{-\alpha}$ with $\alpha=2$ (such as in the ABBM model or the mean field version of

16 From the normalization of the avalanche size distribution $D(s, r, h)$ it follows that $\tau>1$. the RFIM), then at (small enough) finite field sweep rate the corresponding noise "pulse" duration distribution is expected to scale as $D(T, \Omega) \sim T^{2-a(H) C \Omega}$ where $a(H)$ and $C$ are nonuniversal constants $(54 ; 55 ; 56 ;$;57).

If, however, in the adiabatic limit the exponent $\alpha$ is either greater or smaller than 2, then at (small) finite field sweep rate the pulse duration distribution exponent remains the same as in the adiabatic limit. Note that in the case $\alpha<2$ especially, $\Omega$ has to be particularly small in order to be able to still see distinct pulses even at finite field sweep rate, since at higher sweep rate the system will very quickly develop runaway events. In (52; 53) quantitative criteria for the meaning of "small sweeprate" are given. Also, the zero temperature nonequilibrium RFIM and recent variants are used to numerically test the analytic results, which are expected to be applicable to a much larger class of systems with crackling noise (the exact conditions are discussed in $(52 ; 53)$ ). It is found that the results agree well with both simulations and recent experiments on Barkhausen noise in various soft magnetic materials with and without applied stress (with $\alpha<2$ and $\alpha=2$ respectively (11)). A brief review of other, related studies of the effects of finite field sweep rate is also given in (52).

\section{SUBLOOPS AND HISTORY INDUCED CRITICAL BEHAVIOR}

One of the characteristic features of magnetic hysteresis are the subloops, seen as the external field is changed up and down an amount insufficient to saturate the magnetization (figure 4).

\section{A. Return-Point Memory}

Much attention has been paid to modeling these subloops with the Preisach model (58) Preisach models are quite different in spirit to ours: they represent the system as a large number of uncoupled hysteretic twostate domains, and fit the distribution to the observed behavior. They are able to model a hysteretic system if it possesses return-point memory (16), also known as wiping out (59).

Return-point memory states that the magnetization after a subloop rejoins a larger loop (perhaps itself a subloop) equals the magnetization at which the subloop left the outer loop. That is, if the subloop represents an excursion downward from a local maximum external field $H$, then when $H$ returns to its previous maximum the magnetization $M$ returns to its previous value - remembering its previous magnetization on returning, and wiping out all effects of the excursion. Return-point memory states that the subloops in figure 4 should close perfectly, without a gap and without crossing themselves (as observed).

Indeed, our interacting, three-dimensional, disordered models exhibit the return-point memory in an even 
stronger form. Upon rejoining the larger loop, the entire state of the system is microscopically identical to the state it had when it left the larger loop (a microscopic return-point memory, as opposed to a macroscopic memory constraining only the net magnetization). In (23), we showed in great generality that return point memory should be true of any system with

1. A partial ordering of states. Here, one microstate $A$ is "ahead" of another $B, A>B$, if every spin in $A$ is greater than or equal to the corresponding spin in $B$.

2. No passing. That is, the dynamics preserves the partial ordering.

3. Adiabatic. The external field is raised and lowered slowly enough that the system does not lag behind.

Return-point memory can be quite remarkable: in our model (and in some experimental systems) repeating a subloop plays back precisely the same Barkhausen noise. Indeed, the return-point memory can be used as a way of retrieving analogue magnetic memories that is (at least theoretically) significantly superior to measuring the remanent magnetization (60). On the other hand, the absence of exact (microscopic) return point memory has also been observed $(61 ; 62 ; 63)$, and there are various interesting experiments testing for the reproducibility of magnetic avalanches $(64 ; 65 ; 66)$ and for microscopic return point memory and complementary point memory in magnets at various disorders (67) ${ }^{17}$ In (67) the authors experimentally study the influence of disorder on major loop return point memory and complementary return point memory in $\mathrm{Co} / \mathrm{Pt}$ samples with varying interfacial roughness and find with increasing disorder the onset and saturation of both return point memory and complementary point memory.

Disorder dependence of return point memory versus reptation (i.e. is gradual subloop closure (68; 69)) has recently been reported for the zero temperature random coercivity model with antiferromagnetic-like interactions (70).

A related memory effect, recently reported for the hysteresis of spin glasses, is the reversal field memory effect (71). Katzgraber et al. show that this memory effect emerges in the nonequilibrium Edwards Anderson spin glass (EASG) when the magnetic field is first decreased from its saturation value and then increased again from some reversal field $H_{R}$. The authors find that EASG exhibits a singularity at the negative of the reversal field,

17 The complementary point memory relates the magnetic domains at one point on the major hysteresis loop to the domains at the complementary point on the major loop during the same and during subsequent cycles.
$-H_{R}$, in the form of a kink in the reversal of the magnetization of the reversal curve. They show that this memory effect is due to a local spin-reversal symmetry of the Hamiltonian. This symmetry and thus the reversal field memory effect is present in spin glasses where the disorder is due to random couplings between spins. In general it is not expected in systems with random magnetic fields such as the RFIM, since there the disorder breaks local spin reversal symmetry.

As an extension to memory effects for a driving field that varies in two dimensions, a vector form of wipe-out memory for situations when the magnetic field varies in both direction and magnitude, has been suggested based on the two dimensional vector Preisach model in (72), and has been experimentally tested in (73).

\section{B. Critical Behavior in Subloops}
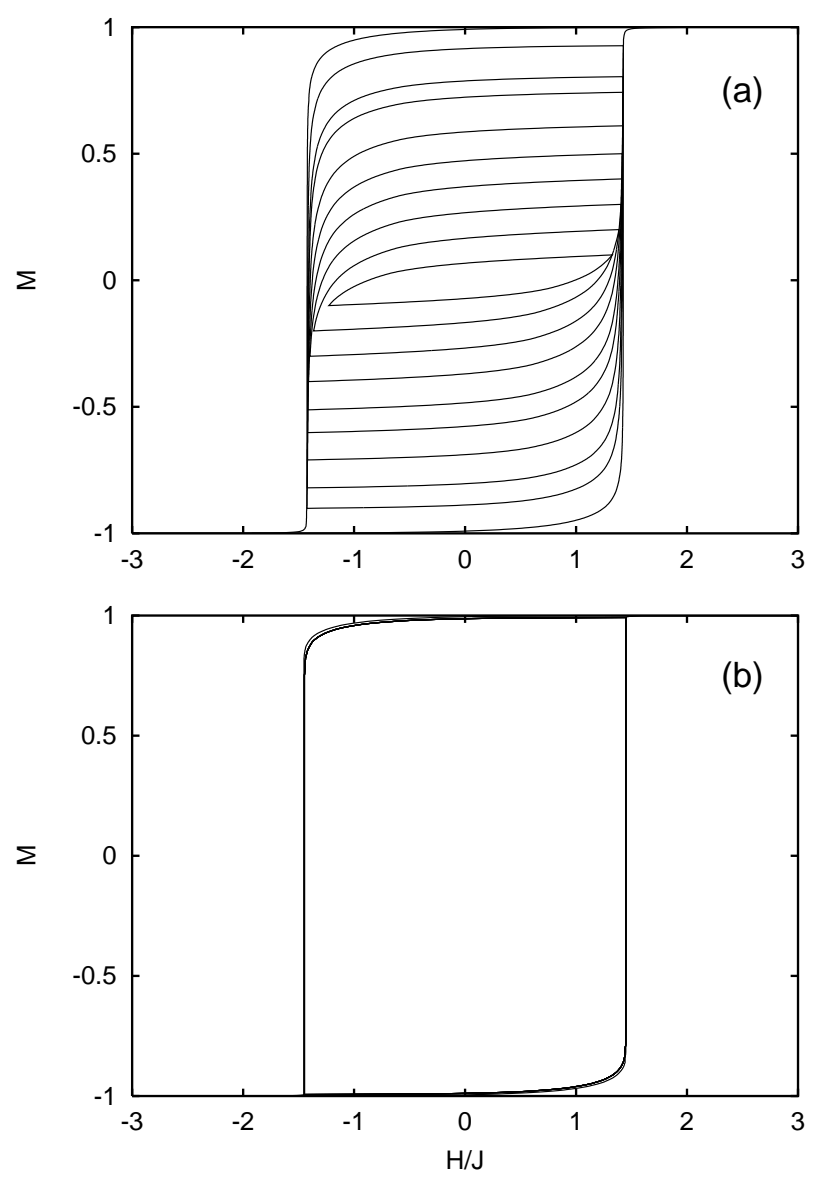

FIG. 22 Saturation hysteresis loops with concentric inner subloops. Top: $R=2.225 \mathrm{~J}>R_{c}$; bottom: $R=$ $2.1<R_{c}$. (74)

The disorder induced critical scaling for hysteresis loops in the RFIM that we discussed so far all referred to the saturation hysteresis loop. However, often it is 
experimentally impractical to take magnets to their saturation point due to the large magnetic fields required, so the behavior of subloops (figure 22. (74)) is of great interest to experiments and applications. The RFIM can be used to model subloops, and in one dimension (75) and on a Bethe lattice $(76$; 77) they have been computed exactly (78). The magnetization curves of subloops have been collapsed near the demagnetized state using Rayleigh's law (12; 79). Interestingly, in the RFIM not only the saturation hysteresis loops but also subloops reflect the critical point described above, and at the critical disorder one finds history-induced critical scaling. Concentric inner subloops resemble rescaled saturation loops at effectively higher (possibly correlated) disorder. Avalanche size distributions for the inner subloops, too, look similar to rescaled avalanche size distributions of saturation loops at higher disorder. We have studied universal properties of this history induced critical behavior using numerical simulations of systems with more than 100,000,000 spins and Widom scaling methods and extracted universal scaling exponents and scaling functions. These predictions are expected to apply to experiments on hysteresis and Barkhausen noise in hard magnetic materials. To model soft magnets on the other hand, we added demagnetizing fields to the model and obtained single domain wall propagation, as described in (13). As a result the system was always critical, even for the subloops. The results of this study are discussed in $(74 ; 80)$. A special, related kind of subloops - so called "first order reversal curves" (FORC) - have proven very useful in distinguishing between different magnetic materials, see (81).

\section{Demagnetization Curves}

The RFIM also predicts disorder induced critical behavior in the demagnetization curve, or initial magnetization curve and the associated Barkhausen noise. Ferromagnetic materials with a remanent magnetization can be demagnetized by applying an oscillating magnetic field with amplitude slowly decreasing from a large initial value to zero. Sometimes the final state is also termed AC-demagnetized state. The oscillating external field with decreasing amplitude takes the system through concentric subloops. The line connecting the tips of the subloops is known as the normal or initial magnetization curve, or demagnetization curve. It is to be distinguished from the virgin curve which is obtained by thermal demagnetization $(16 ; 82 ; 83)$. One can use the RFIM to simulate the demagnetization curve for different amounts of disorder, especially near the critical disorder mentioned above for the saturation loop. It turns out that this curve reflects much of the scaling behavior found for the saturation hysteresis loop. This may be surprising, since the meta-stable states encountered in the demagnetization curve are completely different from those of the saturation loop. In (84) the model is briefly described and the necessary exponent relations are derived, which show that the exponents for the demagnetization curve can actually be derived from those of the subloops. Predictions for the exponents from simulations of up to one million spins are presented. The demagnetization curve in the presence of long range demagnetizing fields is also discussed, with resulting predictions for experiments on demagnetization curves and associated Barkhausen noise in soft magnetic materials. For a discussions of the Rayleigh law and scaling of the demagnetization curve at small magnetic fields, see (77; 79). Zarand, Pazmandi, Pal, and Zimanyi recently proposed a new optimization method to search for ground states, which is based on the demagnetization procedure (85).

\section{REAL EXPERIMENTS}

\section{A. Magnetic hysteresis loops}

There are a few recent experiments on hysteresis and crackling noise where disorder is tuned and evidence of a phase transition related to that of the nonequilibrium RFIM is reported: In (86), Berger et al. describe a transition from smooth magnetic hysteresis loops to those with a jump in the magnetization in ultrathin epitaxial Gd-films with in-plane magnetization upon annealing the sample at successively higher annealing temperatures. The authors show that annealing at higher temperature increasingly reduces the disorder in the crystal structure of the films. In $87 ;$ 88; 89) Berger et al. report a similar transition for thin $\mathrm{Co} / \mathrm{CoO}$ films. In this system the antiferromagnetic $\mathrm{CoO}$ layer allows a reversible tuning of the magnetic disorder felt by the ferromagnetic Co layer, by simple temperature variation. The authors are able to extract scaling collapses for the magnetization hysteresis loops at different disorders, and find the values of the associated scaling exponents to be $\beta=0.022 \pm 0.006$ and $\beta \delta=0.30 \pm 0.03$. Note that this magnetic system is effectively two dimensional.

Incidentally, almost the same exponent values $(\beta=$ $0.03 \pm 0.01$ and $\beta \delta=0.4 \pm 0.1)$ are reported by Marcos et al. in (90) for the magnetic hysteresis loops of $\mathrm{Cu}-\mathrm{Al}$ Mn alloys with different Mn content at low temperatures. The loops are smooth above a certain temperature, but exhibit a jump in the magnetization below that temperature. The authors perform a scaling analysis near this critical point with temperature as the effective "disorder" tuning parameter. They point out that, contrary to the thin $\mathrm{Co} / \mathrm{CoO}$ bilayers in Berger et al. (87), this system is effectively three dimensional.

For these three systems it would be very interesting to also see a scaling analysis of the associated Barkhausen noise, since from simulations we know that the exponents extracted from avalanche size distributions and noise power spectra are often more reliable than those obtained just from magnetization curves alone (27). For these three systems, so far, only the scaling of the mag- 
netization curves has been reported.

\section{B. Disorder effects on Barkhausen Noise}

Disorder effects on crackling noise (rather than hysteresis loops) has been seen in other systems. Barkhausen noise is commonly used for nondestructive testing - in engineering contexts we find careful studies of the effect of the amount of carbon content in steel on Barkhausen noise. One might speculate that varying the carbon content could be similar to varying the effective disorder in the system. It would be interesting to see a systematic scaling analysis for the available experimental data. In an early study (49) Lieneweg and Grosse-Nobis found that the distribution of Barkhausen pulse areas integrated over the hysteresis loop of an $81 \% \mathrm{Ni}-\mathrm{Fe}$ wire was well described by a power law (with exponent $\tau+\sigma \beta \delta$ ranging from 1.73 to 2.1) up to a certain cutoff size. Annealing the sample at various annealing temperatures they found that the cutoff appeared to be smaller at higher annealing temperatures. It would be interesting to see whether the cutoff grows to takes a (system-size dependent) maximum value at a critical annealing temperature $T_{c}^{a n n}$ and decreases again at higher and lower annealing temperatures, as one would expect if the RFIM applies with temperature as the effective disorder tuning parameter in this system. (In leading approximation, near $T_{c}^{a n n}$ they should be linearly related to each other.) Near $T_{c}^{a n n}$ the Barkhausen pulse area distribution should then be described by a scaling form that would allow a scaling collapse of all distributions onto one single curve for appropriate rescaling of the axes. Again, potentially universal critical exponents could be extracted from such a collapse. They would be predicted by the RFIM avalanche critical exponents if that model is in the same universality class.

There are other experiments which revealed power law decays for Barkhausen pulse size distributions in various samples, as reviewed for example in (1) and in the article by Durin and Zapperi in this book (42). Many of these are measurements in soft magnetic materials where long range interactions are important and the main mechanism for Barkhausen noise is domain wall propagation rather than new domain nucleation. Disorder has usually not been varied in these experiments. Models predict that these systems naturally tend to operate close to the underlying critical domain wall depinning transition and thus produce power law pulse size distributions regardless of the underlying disorder, (at least as long as it is less than a critical value above which the domain wall may have overhangs $(5 ; 8 ; 9 ; 10 ; 11 ; 12)$ ). The disorder induced critical point of the nonequilibrium RFIM is expected to be relevant for hard magnetic materials instead, where either the disorder is very strong compared to the effects of long range dipolar interactions, or the geometry of the sample and experiment is chosen such that the effects of long range dipolar fields are small (as for thin films with in-plane applied magnetic field, or for thin wires with parallel applied field).

\section{Imaging magnetic avalanches and states}

The avalanche structures predicted by the theoretical models are visually interesting (figure 23); in our model they have fractal dimensions $1 / \sigma \nu$ close to, but probably less than three. Exciting experiments probing these spatial structures are coming on line.

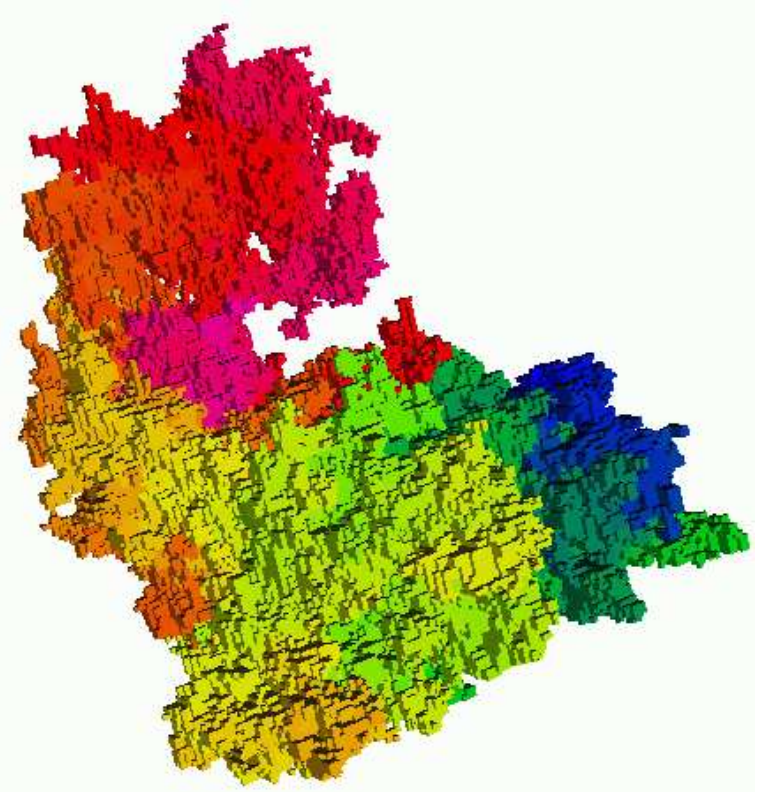

FIG. 23 Fractal spatial structure of an avalanche. This moderate-sized avalanche contains 282,785 domains (1).

Recently interesting experiments imaging avalanches in magnetic films have been reported. In (91) Kim, Choe, and Shin report full-field magneto-optical observations (via Kerr effect) of Barkhausen avalanches in Co polycrystalline thin films at criticality, i.e. their imaged avalanches are due to single domain wall propagation rather than domain nucleation. In this experiment many avalanches extend far beyond the imaged region, so that their measured exponent appears to be an exponent combination of the exponents computed in the models. In addition to their results it'd be interesting to also see time resolved images of entire avalanches and direct measurements of the roughness exponent of the propagating domain wall, to compare with predictions from models for magnetic domain wall propagation. The magnetization reversal dynamics of epitaxial Fe films grown on $\mathrm{GaAs}(001)$ and the field dependent evolution of domain structure at various field has also been studied using the magneto-optic Kerr effect, see for example (92). In (93) Schwarz et al. report on visualization of the Barkhausen effect by magnetic force microscopy for a granular thin 
film with perpendicular anisotropy. The authors are able to localize individual Barkhausen volumes and distinguish them as either newly nucleated or grown by domain wall propagation. They find a Gaussian size distribution of nucleated Barkhausen volumes indicating an uncorrelated random process, and a power law distribution for grown Barkhausen volumes, reflecting critical behavior during domain wall motion. Another very interesting experimental approach is x-ray speckle metrology (67). In a recent preprint Pierce et al use this method to study correlations between microstates with different histories and test for microscopic return point memory of subloops in magnetic systems.

\section{Random Bonds and Random Anisotropy}

Renormalization group treatments, symmetry arguments (27), and simulations $(94 ; 95)$ show, that the hysteretic, zero temperature random bond Ising model with ferromagnetic mean bond strength is expected to be in the same universality class as the nonequilibrium RFIM. Vives et al. also point out that their numerical scaling exponents in two dimensions of the two models also have overlapping error bars with the corresponding exponents of Blume-Emery Griffiths models with disorder, in which the spins take three values $\pm 1,0(95)$. An analysis of the short time dynamics of first-order phase transitions in a disordered system, using the random bond Ising model is given in (96). The diluted ferromagnetic random Ising model (97) has both nonuniform bond strengths that reflect dilution due to nonmagnetic impurities, and random magnetic fields. Symmetry arguments suggest (27) that a model with uncorrelated random (ferromagnetic) bonds and random fields should again be in the same universality class as the RFIM, since it has no new symmetries compared with the RFIM. Similarly, daSilveira and Kardar developed a $O(N)$ vector spin model with random fields and showed through a renormalization group calculation that, in general, it can displays the same continuous phase transition in the hysteresis loop as the RFIM (98), i.e. the two critical points are in the same universality class. ${ }^{18}$ The reason is that the applied external field breaks any added rotational symmetry (27) so that the behavior on long length scales is determined by the same the same symmetries in both models. Similarly the random anisotropy model of (99), was shown numerically to be in the same universality class as the RFIM: this is again as expected, since the external applied field not only breaks rotational symmetry but also time-reversal symmetry. Perhaps somewhat surprisingly, a transition from hysteresis loops with a jump to those without, like the one of the RFIM, is also seen in a plastic depinning model for charge density waves (100). If

18 They also find a new universality class, see section $\mathrm{X}$ in the random field Ising model a certain fraction of the bonds between neighboring spins is enhanced to a very large value, one obtains a simple model for exchange bias (101).

Hysteresis loops observed for the Ising glass with zero ferromagnetic mean bond strength are smooth and continuous $(102 ; 103)$ - qualitatively this agrees with experimental hysteresis loops seen for example in AuFe systems (104). For Ising glasses with strong ferromagnetic mean bond strength (compared to the width of the distribution of bond strengths, which is the disorder), a jump in the magnetization curve appears, just as in the low disorder phase of the RFIM. The hysteresis loops resemble those seen for more concentrated AuFe systems (104) as well as CuMn and AgMn systems (105; 106). A brief review of these and other glassy spin systems such as Ising spin glasses with long-range interactions and Heisenberg spin glasses with long-range interactions in both theory and experiment is given for example in (103).

An interesting hysteresis model with rather different properties than ours based on an infinite-range spin glass (Sherrington-Kirkpatrick model) is reported to show selforganized criticality; see (102) for details.

\section{E. Other dynamics at high sweep rates}

Much interesting work has been done in both theory and experiment on dynamic hysteresis loops at finite field sweep rate in the presence of temperature. At field sweep rates higher than a critical value that depends on sweep amplitude and temperature, the system transitions to a symmetry broken phase with a flatter hysteresis loop, centered around a nonzero average magnetization. Similar dynamic transitions also are seen for pulsed and stochastically varying fields. Chakrabarti and Acharyya published a review article on both models and experiments in this regime (107). Some of the addressed questions deal with scaling of the hysteresis loop area with field sweep frequency, amplitude, and temperature. While in the initial approaches to these issues quenched randomness in the system was neglected, there are recent model approaches and experiments studying dynamic hysteresis of a moving domain wall in the presence of quenched disorder and temperature at finite field sweep rate $(19 ; 21)$.

\section{F. Nonmagnetic noisy hysteretic systems}

In this section we briefly discuss a broad range of related work on avalanches and hysteresis in non-magnetic systems. We start with a general overview, and then focus on a few particular systems. 


\section{Systems with avalanches}

Magnets crackle (Barkhausen noise) when the external field is ramped upward slowly. Many systems exhibit similar crackling noise: when pushed slowly they respond with discrete events of a broad range of sizes. ${ }^{19}$ The earth responds (108) with violent and intermittent earthquakes as two tectonic plates rub past one another. A piece of paper (4) (or a candy wrapper at the movies $(109 ; 110)$ ) emits intermittent, sharp noises as it is slowly crumpled or rumpled. Just like the magnetization avalanches responsible for Barkhausen noise in magnets, these individual events span many orders of magnitude in size. Indeed, the distribution of sizes follows a power law with no characteristic size scale.

In the past decades, scientists in a variety of fields have been making rapid progress in developing models and theories for understanding this sort of scale-invariant behavior in driven, nonlinear, dynamical systems. Interest in these sorts of phenomena goes back several decades. The work of Gutenberg and Richter (108) in the 1940's and 1950's established the well-known frequencymagnitude relationship for earthquakes that bears their names. A variety of many-degree-of-freedom dynamical models (111; 112; 113; 114; 115; 116; 117; 118; 119; 120; 121; 122), with and without disorder, have been introduced in the years since to investigate the nature of slip complexity in earthquakes. More recent impetus for work in this field came from the study of the depinning transition in sliding charge-density wave (CDW) conductors in the 1980's and early 1990's 1 123; 124; 125; 126; 127; 128; 129). Interpretation of the CDW depinning transition as a dynamic critical phenomenon sprung from Fisher's early work (123; 124), and several theoretical and numerical studies followed. This activity culminated in the RG solution by Narayan and Fisher (126) and the numerical studies by Middleton (127) and Myers (128) which combined to provide a clear picture of depinning in CDWs and open the doors to the study of other disordered, nonequilibrium systems.

Bak, Tang, and Wiesenfeld inspired much of the succeeding work on crackling noise (130; 131). They introduced the connection between dynamical critical phenomena and crackling noise, and they emphasized how systems may naturally end up at the critical point through a process of self-organized criticality. (Their original model was that of avalanches in growing sandpiles. Sand has long been used as an example of crackling noise $(132 ; 133)$. However, it turns out that real sandpiles don't crackle at the longest scales $(134 ; 135))$.

Researchers have studied many systems that crackle. Simple models have been developed to study bubbles rearranging in foams as they are sheared (136), biological

19 This subsection follow closely the presentation in reference (1), with some added references. extinctions (137), ${ }^{20}$ fluids invading porous materials and other problems involving invading fronts $(\underline{6} ; 34 ; 35 ; 140$; $141 ; 142),{ }^{21}$ the dynamics of superconductors (143; 144; 145), and superfluids (146; 147), sound emitted during martensitic phase transitions (148), fluctuations in the stock market $(149 ; 150 ; 151)$, solar flares (152), cascading failures in power grids $(153 ; 154 ; 155)$, failures in systems designed for optimal performance (156; 157; 158), group decision making (159), crackling noise in mammalian lungs (160), and fracture in disordered materials $(161 ; 162 ; 163 ; 164 ; 165 ; 166)$. These models are driven systems with many degrees of freedom, which respond to the driving in a series of discrete avalanches spanning a broad range of scales what we are calling crackling noise.

There has been healthy skepticism by some established professionals in these fields to the sometimes grandiose claims by newcomers proselytizing for an overarching paradigm. But often confusion arises because of the unusual kind of predictions the new methods provide. If our models apply at all to a physical system, they should be able to predict all behavior on long length and time scales, independent of many microscopic details $f$ the real world. This predictive capacity comes, however, at a price: our models typically don't make clear predictions of how the real-world microscopic parameters affect the long-length-scale behavior. In this paper and in (1) we have provided overview of the renormalization-group (167; 168; 169; 170; 171) that many researchers use to understand crackling noise and which is perhaps the most impressive use of abstraction in science.

We now turn to some non-magnetic systems that have been particularly well studied.

\section{Martensites}

Hysteresis with crackling noise and in certain cases even microscopic return point memory has long been seen in ferroelastic materials, such as shape memory alloys or martensites $(172 ; 173)$. A martensitic transformation is a diffusionless first order phase transformation where the lattice distortion is mainly described by a homogeneous shear. Many metals and alloys with a bcc structure will upon cooling (or under strain) undergo this transition to a low temperature close packed structure. In athermal martensites, such as $\mathrm{Cu}-\mathrm{Zn}-\mathrm{Al}$, thermal fluctuations are not relevant - temperature acts as an external driving field. Similar to ferromagnets ramping magnetic field, the martensitic transition takes place as a sequence of avalanches as the temperature is swept. The analogue of Barkhausen noise is actual noise: acoustic emission

20 These models are controversial (138; 139): they ignore catastrophic external events like asteroids.

21 The random-field model we describe was invented in this context $(6 ; 140)$, as described in section III 
due to elastic waves in the ultrasonic range, generated by propagating domain walls during the avalanches.

Experimental studies of the acoustic emission generated during a thermally induced martensitic transformation of a $\mathrm{Cu}-\mathrm{Zn}-\mathrm{Al}$ single crystal revealed an apparent absence of characteristic scales in the distribution of avalanches (174). After an initial number of hysteresis cycles (ramping temperature) the system reaches a final attractor yielding two decades of power law scaling in the time distribution of acoustic emission pulse durations. The authors argue that the cutoff at large times is probably due to experimental limitations (amplifier cutoff), and interpret the observed scaling behavior in terms of self-organized criticality. Of course, the system could have a tunable parameter that lies close to the critical point (two decades of power-law scaling arises in a range $50 \%$ away from our critical point).

In a more recent study, Pérez-Reche, Stipcich, Vives, Mañosa, Planes, and Morin (175) study the evolution of the kinetic features of the martensitic transition in a $\mathrm{Cu}$ Al-Mn single crystal under thermal cycling. The authors use several experimental techniques, including optical microscopy, calorimetry, and acoustic emission, to perform an analysis at multiple scales. Focussing especially on avalanches, they find that there are significant differences between the kinetics at large and small scales during the initial cycles. Upon repeated temperature cycles, however, the system evolves from displaying a supercritical avalanche size distribution to a critical power-law distribution. In the language of the RFIM this could be described as the effective disorder being increased by the temperature cycles from $R<R_{c}$ to $R=R_{c}$. (The critical exponents, and thus the universality class, is different from our RFIM).

Both of these systems demand repeated cycling to produce critical behavior. This is quite different from systems with return-point memory, where repeated cycles don't change the behavior. A recently introduced, simple two dimensional phenomenological model for the martensitic transformation is shown to mimic near full reversal of morphology under thermal cycling in (176; 177).

A crossover from the hysteresis loops with a jump or "burst" to smooth hysteresis loops (ramping temperature) has been recorded in (178), as the grain size of macroscopic, polycrystalline specimen of an Fe-Ni-C alloy is reduced. The crossover is explained in terms of finite size effects in defect induced nucleation within each grain (due to extended defects like dislocation tangles, however, rather than random point disorder). It seems clear that one should look for critical fluctuations near the crossover that would promote an interpretation of the grain size as the analogue of the disorder parameter $R$ in our model.

\section{Liquid Helium in Nuclepore}

In (179) Lilly, Finley, and Hallock show a hysteresis loop for the capillary condensation of superfluid helium in the porous material Nuclepore. Nuclepore is a polycarbonate sheet perforated by a high density of nearly cylindrical holes with a nominal diameter of about $2000 \AA$. The pores intersect. The most probable number of intersections per pore is about 5. Most pores participate in a macroscopic interconnected network, (i.e. they are apparently internally connected beyond the percolation threshold). In the experiment the authors measure the amount of helium absorbed and capillary condensed in the Nuclepore sample as a function of the number of helium atoms present (chemical potential) at fixed temperature $T=1.52 \mathrm{~K}$.

Pore intersections result in weak interactions among the pores which lead to avalanches that have been observed upon slow withdrawal of helium from the cell. The upper branch of the hysteresis loop (upon withdrawal of He from the sample) actually has microscopic jumps. The largest avalanche observed involves $2.610^{7}$ pores, which is about $2.6 \%$ of the system. The distribution of avalanche sizes is described by power laws.

This system has clear analogies to our model. The coupling between the pores is certainly "ferromagnetic": If a pore is emptied it becomes more likely for an intersecting pore to be emptied also. Different pore radii and different cross-sections of the pore intersections introduce a certain randomness which might be used to tune the system to a critical point. In (180) the avalanches are studied for different characteristic pore sizes and the revelance of various theoretical models which one might apply to the system is discussed. In (181) a unifying theoretical approach to hysteresis phenomena associated with capillary condensation of gases in disodered mesoporous solids is given, and the connection with the zero-temperature theoretical studies of magnetization-reversal hysteresis of driven interfaces in disordered media is discussed (see also (182)).

\section{Superconductors}

a. Superconducting vortex avalanches In an interesting experiment on the dynamics of superconducting vortices in the Bean state in $N b_{47 \%} T i_{53 \%}$, Field, Witt and Nori recorded vortex avalanches as the system was driven to the threshold of instability by the slow ramping of the external magnetic field (143). The individual avalanches were ranging in size from 50 vortices up to about $10^{7}$ vortices, which is about $10^{-6}$ of the total number of vortices in the system. The distribution of avalanches sizes was recorded for the events occurring in three $450 \mathrm{G}$ wide windows of the external magnetic field range, centered at $2.25 \mathrm{kG}, 5.33 \mathrm{kG}$, and $7.55 \mathrm{kG}$. At all three values for the field $H$ there seems to be a power law up to some cutoff size, which varies with $H$. The authors suggest 
that the self-organized criticality is the underlying mechanism, despite the fact that their cutoff size changes as a function of $H$.

b. Ultra-thin granular superconducting films in a parallel field $\mathrm{Wu}$ and Adams discovered avalanches in film resistance corresponding to the collapse of macroscopic superconducting regions involving up to $10^{9}$ grains near the parallel critical field in ultra-thin granular superconducting Al-films (183). The corresponding distribution of avalanche sizes displays four decades of power law scaling, (which in our simulations would correspond to a value of disorder about 10-15\% away from the critical point). Fewer decades are seen at lower external magnetic fields - the field may act as a tunable parameter for a critical point. The authors point out the relation to our model and the zero temperature random bond Ising model with the same dynamics. The scaling exponent they extract from the power law decay is similar to the prediction from simulations of the two dimensional random bond Ising model.

\section{Prewetting on a disordered substrate}

Blossey, Kinoshita, and Dupont-Roc have studied the effect of substrate randomness on the dynamics of a prewetting transition (184). In their theoretical paper they first review several experiments and then suggest a mapping of the hysteretic prewetting system to a two dimensional nonequilibrium RFIM in an external field $H$, by identifying the Ising spins \pm 1 with a wet or nonwet patch of the surface. They find that the covering of the substrate appears to be a critical transition. The growth exponents that they extract from their simulations are reported to be compatible with those of percolation, see also (185).

\section{UNSOLVED PROBLEMS} field.

There are many open and challenging questions in this

1. Two Dimensions. Except for the experiments in magnets and martensites most of these experiments are in effectively two dimensional systems. $D=2$ appears to be the lower critical dimension of the transition in the RFIM (3; 41). Extracting reliable scaling behavior in two dimensions remains a challenge, and we have focused here on three dimensions where our understanding is reasonably complete.

2. Avalanche average pulse shapes. The average pulse shapes predicted by the various theories (figure 14) do not appear close to those measured in experiments, which themselves disagree with one another (figure 13). This is one of the few experiments where the scaling function has been extracted from the data. Are we mistaken about the universality of these functions? Does this reflect measurement artifacts, or perhaps unrecognized important control parameters? Are there analytical corrections to scaling (appendix $\mathrm{A}$ ) that we are missing that skew the curves? Or are there many dynamical universality classes? Recent experiments (186) suggest that the skewness of the smaller avalanches can be affected by external stress. Signatures of time asymmetries have also been seen in high order spectra studies of Barkhausen Noise (187; 188).

3. Correlated disorder and long-range fields. Many of the experiments involve long-range fields which may in principle alter the universality class perhaps in ways that differ from that of the infiniterange model. Some experiments involve different kinds of disorder, such as correlated disorder rather than point disorder and random anisotropies or random bonds rather than random fields (which probably will not change the universality class). Also, there are new possible critical behaviors when one starts with other local order parameters: for example, if we start not with Ising spins but with vectors (98).

\section{Extracting exponents and scaling functions.} We need a more organized approach, both experimentally and computationally, for extracting critical exponents and reporting scaling functions. These hysteresis models represent a serious challenge, far more difficult than most of the critical points studied in equilibrium phase transitions. In part this is because the critical behavior is so much more important: because the avalanches become large so far from $R_{c}$, one cannot get close to $R_{c}$ without having severe finite size effects - hence the $R$ dependence of the parameters becomes a problem.

\section{Acknowledgments}

The work (and many of the discussions) reviewed in this article represent collaborations with many of our students and colleagues. We acknowledge support from NSF DMR-0218475, NSF DMR-0314279 and NSF DMR9976550 (Materials Computation Center). 


\section{APPENDIX A: Derivation of the scaling forms and corrections}

In our work we make extensive use of scaling collapses. $^{22}$ Many variations are important to us: Widom scaling, finite-size scaling, singular corrections to scaling, analytic corrections to scaling, rotating axes, and exponentially diverging correlation length scaling. The underlying theoretical framework for scaling is given by the renormalization group, in the context of equilibrium critical phenomena and by now well explicated in a variety of texts.

We have discovered that we can derive all the scaling forms and corrections that have been important to us from two simple hypotheses (found in critical regions): universality and invariance under reparameterizations. Universality is the statement that two completely different systems will behave the same near their critical point (for example, they can have exactly the same kinds of correlations). Reparameterization invariance is the statement that smooth changes in the units or methods of measurement should not affect the critical properties or rather, that the predictions of our scaling theories are only valid up to a smooth change of coordinates in the control parameters (the analytic corrections to scaling we discussed in section (I). We use these properties to develop the scaling forms and corrections we use in this paper. Each example we cover will build on the previous ones while developing a new idea.

Our first example repeats our discussion of size versus duration in section IV Consider some property $F$ of a system at its critical point, as a function of a scale $x$. $F$ might be the spin-spin correlation function as a function of distance $x$ (or it might be the avalanche probability distribution as a function of size $x$, etc.) If two different experimental systems are at the same critical point, their $F$ 's must agree. It would seem clear that they cannot be expected to be equal to one another: the overall scale of $F$ and the scale of $x$ will depend on the microscopic structure of the materials. The best one could imagine would be that

$$
F_{1}\left(x_{1}\right)=A F_{2}\left(B x_{2}\right)
$$

where $A$ would give the ratio of, say, the squared magnetic moment per domain of the two materials, and $B$ gives the ratio of the domain sizes.

Now, consider comparing a system with itself, but with a different measuring apparatus. Universality in this selfreferential sense would imply $F(x)=A F(B x)$, for suitable $A$ and $B$. If instead of using finite constant $A$ and $B$, we arrange for an infinitesimal change in the measurement of length scales, we find:

$$
F(x)=(1-\alpha \epsilon) F((1-\epsilon) x)
$$

22 This appendix follows closely the presentation in (43). where $\epsilon$ is small and $\alpha$ is some constant. Taking the derivative of both sides with respect to $\epsilon$ and evaluating it at $\epsilon=0$, we find $-\alpha F=x F^{\prime}$, so

$$
F(x) \sim x^{-\alpha} .
$$

The function $F$ is a power-law! The underlying reason why power-laws are seen at critical points is that power laws look the same at different scales.

Now consider a new measurement with a distorted measuring apparatus. Now $F(x) \sim \mathcal{A}[F(\mathcal{B}(x))]$ where $\mathcal{A}$ and $\mathcal{B}$ are some nonlinear functions. For example, one might measure the number of microscopic domains $x$ flipped in an avalanche, or one might measure the total acoustic power $\mathcal{B}(x)$ emitted during the avalanche; these two "sizes" should roughly scale with one another, but nonlinear amplifications will occur while the spatial extent of the avalanche is small compared to the wavelength of sound emitted: we expand $\mathcal{B}(x)=B x+b_{0}+b_{1} / x+\ldots$ Similarly, our microphone may be nonlinear at large sound amplitudes, or the absorption of sound in the medium may be nonlinear: $\mathcal{A}(F)=A F+a_{2} F^{2}+\ldots$ So,

$$
\begin{aligned}
& \mathcal{A}[F(\mathcal{B}(x))] \approx \\
& A\left(F(B x)+F^{\prime}(B x)\left(b_{0}+b_{1} / x+\ldots\right)+\right. \\
& \left.\quad F^{\prime \prime}(B x)(\ldots)+\ldots\right)+a_{2} F^{2}(B x)+\ldots
\end{aligned}
$$

We can certainly see that our assumption of universality cannot hold everywhere: for large $F$ or small $x$ the assumption of reparameterization invariance (A4) prevents any simple universal form. Where is universality possible? We can take the power-law form $F(x) \sim x^{-\alpha}=$ $x^{\log A / \log B}$ which is the only form allowed by linear reparameterizations and plug it into (A4), and we see that all these nonlinear corrections are subdominant (i.e., small) for large $x$ and small $F$ (presuming $\alpha>0$ ). If $\alpha>1$, the leading correction is due to $b_{0}$ and we expect $x^{-\alpha-1}$ corrections to the universal power law at small distances; if $0<\alpha<1$ the dominant correction is due to $a_{2}$, and we expect corrections of order $x^{-2 \alpha}$. Thus our assumptions of universality and reparameterization invariance both lead us to the power-law scaling forms and inform us as to some expected deviations from these forms. Notice that the simple rescaling led to the universal powerlaw predictions, and that the more complicated nonlinear rescalings taught us about the dominant corrections: this will keep happening with our other examples.

For our second example, let us consider a property $K$ of a system, as a function of some external parameter $R$, as we vary $R$ through the critical point $R_{c}$ for the material (so $r=R-R_{c}$ is small). $K$ might represent the second moment of the avalanche size distribution, where $R$ would represent the value of the randomness; alternatively $K$ might represent the fractional change in magnetization $\Delta M$ at the infinite avalanche ... If two different experimental systems are both near their critical 
points ( $r_{1}$ and $r_{2}$ both small), then universality demands that the dependence of $K_{1}$ and $K_{2}$ on "temperature" $R$ must agree, up to overall changes in scale. Thus, using a simple linear rescaling $K(r)=(1-\mu \epsilon) K((1-\epsilon) r)$ leads as above to the prediction

$$
K(r)=r^{-\mu} .
$$

Now let us consider nonlinear rescalings, somewhat different than the one discussed above. In particular, the nonlinearity of our measurement of $K$ can be dependent on $r$. So,

$$
\mathcal{A}_{r}(K(r))=a_{0}+a_{1} r+a_{2} r^{2}+\ldots+a_{01} K(r)+\ldots
$$

If $\mu>0$, these analytic corrections don't change the dominant power law near $r=0$. However, if $\mu<0$, all the terms $a_{n}$ for $n<-\mu$ will be more important than the singular term! Only after fitting them to the data and subtracting them will the residual singularity be measurable. For the fractional change in magnetization: $\Delta M \sim r^{\beta}$ has $0<\beta<1$ (at least above three dimensions), so we might think we need to subtract off a constant term $a_{0}$, but $\Delta M=0$ for $R \geq R_{c}$, so $a_{0}$ is zero. On the other hand, in a previous paper(27), we discussed the singularity in the area of the hysteresis loop: Area $\sim r^{2-\alpha}$, where $2-\alpha=\beta+\beta \delta$ is an analogue to the specific heat in thermal systems. Since $\alpha$ is near zero (slightly positive from our estimates of $\beta$ and $\delta$ in 3, 4, and 5 dimensions), measuring it would necessitate our fitting and subtracting three terms (constant, linear, and quadratic in $r$ ): we did not measure the area for that reason.

For our third example, let's consider a function $F(x, r)$, depending on both a scale $x$ and an external parameter $r$. For example, $F$ might be the probability $D_{\text {int }}$ that an avalanche of size $x$ will occur during a hysteresis loop at disorder $r=R-R_{c}$. Universality implies that two different systems must have the same $F$ up to changes in scale, and therefore that $F$ measured at one $r$ must have the same form as if measured at a different $r$. To start with, we consider a simple linear rescaling:

$$
F(x, r)=(1-\alpha \epsilon) F((1-\epsilon) x,(1+\zeta \epsilon) r) .
$$

Taking the derivative of both sides with respect to $\epsilon$ gives a partial differential equation that can be manipulated to show $F$ has a scaling form. Instead, we change variables to a new variable $y=x^{\zeta} r$ (which satisfies $y^{\prime}=y$ to order $\epsilon)$. If $\tilde{F}(x, y) \equiv F(x, r)$ is our function measured in the new variables, then

$$
F(x, r)=\tilde{F}(x, y)=(1-\alpha \epsilon) \tilde{F}((1-\epsilon) x, y)
$$

and $-\alpha \tilde{F}=x \partial \tilde{F} / \partial x$ shows that at fixed $y, F \sim x^{-\alpha}$, with a coefficient $\mathcal{F}(y)$ which can depend on $y$. Hence we get the scaling form

$$
F(x, r) \sim x^{-\alpha} \mathcal{F}\left(x^{\zeta} r\right) .
$$

This is just Widom scaling. The critical exponents $\alpha$ and $\zeta$, and the scaling function $\mathcal{F}\left(x^{\zeta} r\right)$ are universal (two different systems near their critical point will have the same critical exponents and scaling functions). We don't need to discuss corrections to scaling for this case, as they are similar to those discussed above and below (and because none were dominant in our cases).

Notice that if we sit at the critical point $r=0$, the above result reduces to equation (A3) so long as $\mathcal{F}(0)$ is not zero or infinity. If, on the other hand, $\mathcal{F}(y) \sim y^{n}$ as $y \rightarrow 0$, the two-variable scaling function gives a singular correction to the power-law near the critical point: $F(x, r) \sim x^{-\alpha} \mathcal{F}\left(x^{\zeta} r\right) \sim x^{-\alpha+n \zeta}$ for $x<<r^{-1 / \zeta}$ : only when $x \sim r^{-1 / \zeta}$ will the power-law $x^{-\alpha}$ be observed. This is what happened in two dimensions to the integrated avalanche size distribution and the avalanche correlation functions (figures 35,36 , and $37 \mathrm{~b}$ in reference (43)).

For the fourth example, we address finite-size scaling of a property $K$ of the system, as we vary a parameter $r$. If we measure $K(r, L)$ for a variety of sizes $L$ (say, all with periodic boundary conditions), we expect (in complete analogy to (A9))

$$
K(r, L) \sim r^{-\mu} \mathcal{K}\left(r L^{1 / \nu}\right) .
$$

Now, suppose our "thermometer" measuring $r$ is weakly size-dependent, so the measured variable is $\mathcal{C}(r)=r+$ $c / L+c_{2} / L^{2}+\ldots$ The effects on the scaling function is

$$
\begin{gathered}
K(\mathcal{C}(r), L) \sim r^{-\mu} \times \\
\left(\mathcal{K}\left(r L^{1 / \nu}\right)+\right. \\
\left.\left(c L^{1 / \nu-1}+c_{2} L^{1 / \nu-2}\right) \mathcal{K}^{\prime}\left(r L^{1 / \nu}\right)+\ldots\right) .
\end{gathered}
$$

In two and three dimensions, $\nu>1$ and these correction terms are subdominant. In four and five dimensions, we find $1 / 2<\nu<1$, so we should include the term multiplied by $c$ in equation (A11). However, we believe this first term is zero for our problem. For a fixed boundary problem (all spins "up" at the boundary) with a first order transition, there is indeed a term like $c / L$ in $r(L)$. At a critical transition, the leading correction to $r(L)$ can be $c / L$ or a higher power of $L\left(1 / L^{2}\right.$ and so on). This seems to depend on the model studied, the geometry of the system, and the boundary conditions (free, periodic, ferromagnetic, ...) Furthermore, for the same kind of model, the coefficient $c$ itself depends on the geometry and boundary conditions, and it can even vanish, which leaves only higher order corrections. In a periodic boundary conditions problem like ours, we expect that the correction is smaller than $c / L$. Our finite-size scaling collapses for spanning avalanches $N$, the second moments $\left\langle S^{2}\right\rangle$, and the magnetization jump $\Delta M$, were successfully done by letting $c=0$.

For the fifth example, consider a property $K$ depending on two external parameters: $r$ (the disorder for example) 
and $h$ (could be the external magnetic field $H-H_{c}$ ). Analogous to (A9), $K$ should then scale as

$$
K(r, h) \sim r^{-\mu} \mathcal{K}\left(h / r^{\beta \delta}\right) .
$$

Consider now the likely dependence of the field $h$ on the disorder $r$. A typical system will have a measured field which depends on the randomness: $\tilde{\mathcal{C}}(h)=h+b r+b_{2} r^{2}+$ ... (Corresponding nonlinearities in the effective value of $r$ are subdominant.) This system will have

$$
\begin{aligned}
& K(r, \tilde{\mathcal{C}}(h))=r^{-\mu} \times \\
& \quad\left(\mathcal{K}\left(h / r^{\beta \delta}\right)+\left(b r+b_{2} r^{2}\right) r^{-\beta \delta} \mathcal{K}^{\prime}\left(h / r^{\beta \delta}\right)\right)(
\end{aligned}
$$

Now, for our system $1<\beta \delta<2$ for dimensions three and above. This means that the term multiplied by $b$ is dominant over the critical scaling singularity: unless one shifts the measured $h$ to the appropriate $h^{\prime}=h+b r$, the curves will not collapse (e.g., the peaks will not line up horizontally). We measure this (non-universal) constant for our system using the derivative of the magnetization with field $d M / d H(r, h)$. The magnetization $M(r, h)$ and the correlation length $\xi(r, h)$ should also collapse according to equation (A12) (but with $h+b r$ instead of $h$ ); we don't directly measure the correlation length, and the collapse of $M(r, h)$ in the bottom figure 15 includes the effects of the tilt $b$. In two dimensions, $\beta \delta$ is large (probably infinite), so in principle we should need an infinite number of correction terms: in practice, we tried lining up the peaks in the curves (with no correction terms); because we did not know $\beta$ (which we usually obtained from $\Delta M$, which gives $\beta / \nu=0$ in two dimensions), we failed to extract reliable exponents in two dimensions from $d M / d H$.

For the sixth example, suppose $F$ depends on $r, h$, and a size $x$. Then from the previous analysis, we expect

$$
F(x, r, h) \sim x^{-\alpha} \mathcal{F}\left(x^{\zeta} r, h / r^{\beta \delta}\right) .
$$

Notice that universality only removes one variable from the scaling form. One could in practice do two-variable scaling collapses (and we believe someone has probably done it), but for our purposes these more general scaling forms are used by fixing one of the variables. For example, we measure the avalanche size distribution at various values of $h$ (binned in small ranges), at the critical disorder $r=0$. We can make sense of equation (A14 by changing variables from $h / r^{\beta \delta}$ to $x^{\zeta \beta \delta} h$ :

$$
F(x, r, h) \sim x^{-\alpha} \tilde{\mathcal{F}}\left(x^{\zeta} r, x^{\zeta \beta \delta} h\right) .
$$

Before we can set $r=0$, we must see what are the possible corrections to scaling in this case. If the disorder $r$ depends on the field, then instead of the variable $r$, we must use $r+a h$ (the analysis is analogous to the one in example five; other corrections are subdominant). Setting $r=0$ now, leaves $F$ dependent on its first variable, as well as the second:

$$
F(x, r, h) \sim x^{-\alpha} \tilde{\mathcal{F}}\left(x^{\zeta}(a h), x^{\zeta \beta \delta} h\right) \approx x^{-\alpha} \times
$$

$$
\begin{aligned}
& \left(\tilde{\mathcal{F}}\left(0, x^{\zeta \beta \delta} h\right)+\right. \\
& \left.\quad a h x^{\zeta} \tilde{\mathcal{F}}^{(1,0)}\left(0, x^{\zeta \beta \delta} h\right)\right),
\end{aligned}
$$

where $\tilde{\mathcal{F}}^{(1,0)}$ is the derivative of $\tilde{\mathcal{F}}$ with respect to the first variable (keeping the second fixed).

For the binned avalanche size distribution, $x^{\zeta}$ is $S^{\sigma}$, where $0 \leq \sigma<1 / 2$ as we move from two dimensions to five and above. Thus, the correction term will only be important for rather large avalanches, $S>h^{-1 / \sigma}$, so long as we are close to the critical point. Expressed in terms of the scaling variable, important corrections to scaling occur if the scaling variable $X=S^{\sigma \beta \delta} h>h^{1-\beta \delta}$. For us, $\beta \delta>3 / 2$, and we only use fields near the critical field $(h<0.08)$, so the corrections will become of order one when $X=4$ for the largest $h$ we use. In 3 and 4 dimensions, this correction does not affect our scaling collapses, while in 5 dimensions some of the data needs this correction. We have tried to avoid this problem (since we don't measure our data such that it can be used in a twovariable scaling collapse) by concentrating on collapsing the regions in our data curves where this correction is negligible.

A similar analysis can be done for the avalanche time distribution, which has two "sizes" $S$ and $t$ and one parameter $r$ which is set to zero; because we integrate over the field $h$ the correction in A16 does not occur, and other scaling corrections are small.

Finally, we discuss the unusual exponential scaling forms we developed to collapse our data in two dimensions. If we assume that the critical disorder $R_{c}$ is zero and that the linear term in the rescaling of $r$ vanishes ( $\zeta \epsilon r$ in equation (A7) vanishes), then from symmetry the correction has to be cubic, and equation (A7) becomes:

$$
F(x, r)=(1-\alpha \epsilon) F\left((1-\epsilon) x,\left(1+k \epsilon r^{2}\right) r\right)
$$

with $k$ (which is not universal) and $\alpha$ constants, and $\epsilon$ small.

Taking the derivative of both sides with respect to $\epsilon$ and setting it equal to zero gives a partial differential equation for the function $F$. To solve for $F$, we do a change of variable: $(x, r) \rightarrow(x, y)$ with $y=$ $x e^{-a^{*} / r^{2}}$. The constant $a^{*}$ is determined by requiring that $y$ rescales onto itself to order $\epsilon$ : we find $a^{*}=1 / 2 k$. We then have:

$$
0=-\alpha \tilde{F}(x, y)-\frac{\partial \tilde{F}}{\partial x} x
$$

which gives

$$
F(x, r)=x^{-\alpha} \tilde{\mathcal{F}}\left(x e^{-1 / 2 k r^{2}}\right) .
$$

This is one of the forms we use in 2 dimensions for the scaling collapse of the second moments $\left\langle S^{2}\right\rangle_{i n t}$, the avalanche size distribution $D_{\text {int }}$ integrated over the field $H$, the avalanche correlation $G_{i n t}$, and the spanning 
avalanches $N$. We use another form too which is obtained by assuming that the critical disorder $R_{c}$ is not zero but that the linear term in the rescaling of $r$ still vanishes. Instead of equation (A17), we have:

$$
F(x, r)=(1-\alpha \epsilon) F((1-\epsilon) x,(1+\ell \epsilon r) r) .
$$

The function $F$ becomes:

$$
F(x, r)=x^{-\alpha} \tilde{\mathcal{F}}\left(x e^{-1 / \ell r}\right) .
$$

The corrections to scaling for the last two forms (equations A19 and (A21) are similar to the ones discussed above. They are all are subdominant.

\section{References}

[1] Sethna, J. P., Dahmen, K. A., and Myers, C. R. (2001). Crackling Noise. Nature 410, 242.

[2] Mehta, A., Mills, A. C., Dahmen, K. A., and Sethna, J. P. (2002). Universal pulse shape scaling function and exponents: Critical test for avalanche models applied to Barkhausen noise. Phys. Rev. E 65, 046139/1-6.

[3] Perković, O., Dahmen, K., and Sethna, J. P. (1995). Avalanches, Barkhausen Noise, and Plain Old Criticality. Phys. Rev. Lett. 75, 4528.

[4] Houle, P. A., and Sethna, J. P. (1996). Acoustic Emission from Crumpling Paper. Phys. Rev. E 54, 278.

[5] Ji, H., and Robbins, M. O. (1992). Percolative, selfaffine, and faceted domain growth in random 3dimensional magnets. Phys. Rev. B 46, 14519.

[6] Koiller, B., and Robbins, M. O. (2000). Morphology transitions in three-dimensional domain growth with Gaussian random fields. Phys. Rev. B 62, 5771-5778, and references therein.

[7] Cizeau, P., Zapperi, S., Durin, G., and Stanley, H. (1997). Dynamics of a Ferromagnetic Domain Wall and the Barkhausen Effect. Phys. Rev. Lett. 79, 4669.

[8] Urbach, J. S., Madison, R. C., and Markert, J. T. (1995). Interface depinning, self-organized criticality, and the Barkhausen effect. Phys. Rev. Lett. 75, 276.

[9] Narayan, O. (1996). Self-similar Barkhausen noise in magnetic domain wall motion. Phys. Rev. Lett. 77, 3855-3857.

[10] Zapperi, S., Cizeau, P., Durin, G., and Stanley, E. H. (1998). Dynamics of a ferromagnetic domain wall: Avalanches, depinning transition, and the Barkhausen effect. Phys. Rev. B 58, 6353-6366.

[11] Durin, G., and Zapperi, S. (2000). Scaling exponents for Barkhausen avalanches in polycrystalline and amorphous ferromagnets. Phys. Rev. Lett. 84, 4705-4708.

[12] Dante, L., Durin, G., Magni, A., and Zapperi, S. (2002). Low-field hysteresis in disordered ferromagnets. Phys. Rev. B 65, 144441.

[13] Kuntz, M., and Sethna, J. P. (2000). Noise in disordered systems: The power spectrum and dynamics exponents in avalanche models. Phys. Rev. B 62, 11699.

[14] Sides, S., Rickvold, P., and Novotny, M. (1998). Kinetic Ising model in an oscillating field: Finite-size scaling at the dynamic phase transition. Phys. Rev. Lett. 81, 834-837, and references therein.
[15] G.Korniss, White, C., Rikvold, P., and Novotny, M. (2001). Dynamic Phase Transition, Universality, and Finite-Size Scaling in the Two-dimensional Kinetic Ising Model in an Oscillating Field. Phys. Rev. E 63, 016120, and references therein.

[16] Bertotti, G. (1998). Hysteresis in Magnetism. Academic Press.

[17] Roters, L., Lubeck, S., and Usadel, K. (2002). The depinning transition of a driven interface in the random field Ising model around the upper critical dimension. Phys. Rev. E 2002, 026127, 069901(E), and references therein.

[18] Middleton, A. (1992). Thermal rounding of the chargedensity-wave depinning transition. Phys. Rev. B 45, 9465-9468, and references therein.

[19] Nattermann, T., and Pokrovsky, V. (2004). Hysteresis mediated by a domain wall motion. Physica A 340, 625635 , and references therein.

[20] Glatz, A., Nattermann, T., and Pokrovsky, V. (2003). Domain wall depinning in random media by ac fields. Phys. Rev. Lett. 90, 047201, and references therein.

[21] Nattermann, T., Pokrovsky, V., and Vinokur, V. M. (2001). Hysteretic dynamics of domain walls at finite temperatures. Phys. Rev. Lett. 87, 197005.

[22] White, R., Dahmen, K., and Sethna, J. (work in progress).

[23] Sethna, J. P., Dahmen, K. A., Kartha, S., Krumhansl, J. A., Roberts, B. W., and Shore, J. D. (1993). Hysteresis and Hierarchies: Dynamics of Disorder-Driven First-Order Phase Transformations. Phys. Rev. Lett. 70, 3347.

[24] Magni, A. (1999). Hysteresis properties at zero temperature in the dipolar random-field Ising model. Phys. Rev. B 59, 985-990.

[25] Alessandro, B., Beatrice, C., Bertotti, G., and Montorsi, A. (1990). Domain-wall dynamics and Barkhausen effect in metallic ferromagnetic materials. 2. Experiments. J. Appl. Phys. 68, 2908-2915.

[26] Alessandro, B., Beatrice, C., Bertotti, G., and Montorsi, A. (1990). Domain-wall dynamics and Barkhausen effect in metallic ferromagnetic materials. 1. Theory. $J$. Appl. Phys. 68, 2901-2907.

[27] Dahmen, K., and Sethna, J. P. (1996). Hysteresis, Avalanches, and Disorder Induced Critical Scaling: A Renormalization Group Approach. Phys. Rev. B 53, 14872.

[28] Perković, O., Dahmen, K., and Sethna, J. (1999). Disorder-Induced Critical Phenomena in Hysteresis: Numerical Scaling in Three and Higher Dimensions. Phys. Rev. B 59, 6106.

[29] Martin, P. C., Siggia, E., and Rose, H. (1973). Statistical dynamics of classical systems. Phys. Rev. A 8, 423-437.

[30] Bausch, R., Janssen, H., and Wagner, H. (1976). Renormalized field theory of critical dynamics. Z. Phys. B 24, 113-27.

[31] Dominicis, C. D. (1978). Dynamics as a substitute for replicas in systems with quenched random impurities. Phys. Rev. B 18, 4913-4919.

[32] Sompolinsky, H., and Zippelius, A. (1982). Relaxational dynamics of the Edwards-Anderson model and the mean-field theory of spin-glasses. Phys. Rev. B 25, 6860-6875.

[33] Zippelius, A. (1984). Critical dynamics of spin-glasses. 
Phys. Rev. B 29, 2717-2723.

[34] Nattermann, T., Stepanow, S., Tang, L., and Leschhorn, H. (1992). Dynamics of interface depinning in a disordered medium. J. de Physique II 2, 1483-8.

[35] Narayan, O., and Fisher, D. (1993). Threshold critical dynamics of driven interfaces in random media. Phys. Rev. B 48, 7030-42.

[36] Narayan, O. (2000). Anomalous scaling in depinning transitions. Phys. Rev. E 62, R7563-R7566.

[37] Feldman, D. (2002). Critical Exponents of the RandomField O(N) Model. Phys. Rev. Lett. 88, 177202/1-4.

[38] Brezin, E., and Dominicis, C. D. (1998). New Phenomena in the random field Ising model. Europhys. Lett. 44, $13-19$.

[39] Brezin, E., and Dominicis, C. D. (1999). Dynamics versus replicas in the random field Ising model. Comptes Rendus del Academie des Sciences Serie II Fascicule BMecanique Physique Astronomie 327, 383-390.

[40] Spasojevic, D., Bukvic, S., Milosevic, S., and Stanley, H. E. (1996). Barkhausen noise: Elementary signals, power laws, and scaling relations. Phys. Rev. E 54, 2531-2546.

[41] Kuntz, M. (1999). Barkhausen noise: simulations, experiments, power spectra, and two dimensional scaling. Ph.D. thesis, Cornell University.

[42] Durin, G., and Zapperi, S. (2004). The Barkhausen effect. In Science of Hysteresis, Vol. II (G. Bertotti, and I. Mayergoyz, eds.), Elsevier, London.

[43] Perković, O., Dahmen, K., and Sethna, J. P. (1996). Disorder-Induced Critical Phenomena in Hysteresis: A Numerical Scaling Analysis. http://arXiv.org/condmat/9609072.

[44] Sethna, J. P. (work in progress).

[45] Dahmen, K., and Sethna, J. P. (1993). Hysteresis Loop Critical Exponents in 6-epsilon Dimensions. Phys. Rev. Lett. 71, 3222-5.

[46] Pérez-Reche, F., and Vives, E. (2003). Finite-size scaling analysis of the avalanches in the three-dimensional Gaussian random-field Ising model with metastable dynamics. Phys. Rev. B 67, 134421/1-16.

[47] Vives, E., and Pérez-Reche, F. (2004). Avalanche properties of the 3d-RFIM. Physica B 343, 281-285.

[48] Illa, X., and Vives, E. (2004). Statistical properties of pinning fields in the 3d Gaussian RFIM. Physica B 343, 308-313.

[49] Lieneweg, U., and Grosse-Nobis, W. (1972). Distribution of size and duration of Barkhausen pulses and energy spectrum of Barkhausen noise investigated on $81 \%$ nickel-iron after heat treatment. Int. J. Magn. 3, 11-16.

[50] Dahmen, K. A. (1995). Hysteresis, Avalanches, and Disorder Induced Critical Scaling: A Renormalization Group Approach. Ph.D. thesis, Cornell University.

[51] Middleton, A., and Fisher, D. (2002). Threedimensional random-field Ising magnet: Interfaces, scaling, and the nature of states. Phys. Rev. B 65, $134411 / 1-31$.

[52] White, R. A., and Dahmen, K. A. (2003). Driving rate effects on crackling noise. Phys. Rev. Lett. 91, 085702.

[53] Travesset, A., White, R. A., and Dahmen, K. A. (2002). Crackling noise, power spectra, and disorder-induced critical scaling. Phys. Rev. B 66, 024430.

[54] Durin, G., Bertotti, G., and Magni, A. (1995). Fractals, Scaling, and the Question of Self-Organized Criticality in Magnetization Processes. Fractals 3, 351-370.
[55] Bertotti, G., Durin, G., and Magni, A. (1994). Scaling aspects of domain-wall dynamics and Barkhausen effect in ferromagnetic materials. J. Appl. Phys. 75, 5490.

[56] Durin, G. (1997). Thermal magnetic noise with $1 / f$ shape in ferromagnetic alloys. In Proceedings of the 14th International Conference on Noise in Physical Systems and 1/f Fluctuations (C. Claeys, and E. Simoen, eds.), p. 83, World Scientific, Singapore.

[57] Durin, G., Cizeau, P., Zapperi, S., and Stanley, H. (1998). New elements for a theory of the Barkhausen effect. J. Phys. IV 8, 319-322.

[58] Mayergoyz, I. (1991). Mathematical Models of Hysteresis. Springer-Verlag, Berlin.

[59] Mayergoyz, I. (1986). Mathematical Models of Hysteresis. Phys. Rev. Lett. 56, 1518-1521.

[60] Perković, O., and Sethna, J. P. (1997). Improved magnetic information storage using return-point memory. J. Appl. Phys. 81, 1590-1597.

[61] Torre, E. D., and Vajda, F. (1995). Properties of Accommodation Models. IEEE Trans. Mag. 31, 1775.

[62] Guilmart, J. (1970). Reproducibility of Barkhausen Ferromagnetic Noise. Z. Angew. Phys. 28, 266.

[63] Zapperi, S., and Durin, G. (2001). New perspectives for the Barkhausen effect. Comp. Mat. Sci. 20, 436.

[64] Urbach, J. S., Madison, R. C., and Markert, J. T. (1995). Reproducibility of Magnetic Avalanches in an Fe-Ni-Co Ferromagnet. Phys. Rev. Lett. 75, 4694-4697.

[65] Petta, J., Weissman, M., and Durin, G. (1997). Dependence of Barkhausen pattern reproducibility on hysteresis loop size. Phys. Rev. E 56, 2776-2780.

[66] Petta, J., Weissman, M., and O'Brien, K. (1996). Multiple magnetization paths in Barkhausen noise. Phys. Rev. E 54, R1029-R1031.

[67] Pierce, M. S., Buechler, C. R., Sorensen, L. B., Jagler, E. A., Turner, J. J., Kevan, S. D., Chesnel, K. M., Kortright, J. B., Hellwig, O., Fullerton, E. E., Davies, J. E., Liu, K., and Dunn, J. H. (2005). Disorder-induced microscopic magnetic memory. Phys. Rev. Lett. 94, 017202/1-4.

[68] Neel, L. (1958). Sur les effets d'un couplage entre grains ferromagnetiques doues d'hysteresis. Compt. rend. (Paris) 246, 2313.

[69] Neel, L. (1959). Sur les effets des interactions entre les domaines elementaires ferromagnetiques - bascule et reptation. J. Phys. radium 20, 215-221.

[70] Hovorka, O., and Friedman, G. (2003). Onset of reptations and critical hysteretic behavior in disordered systems. http://arXiv.org/cond-mat/0306300 .

[71] Katzgraber, H., Pázmándi, E., Pike, C., Liu, K., Scalettar, R., and Zimanyi, K. V. G. (2002). Reversal Field Memory in the Hysteresis of Spin Glasses. Phys. Rev. Lett. 89, 257202/1-4.

[72] Friedman, G. (1999). Conditions for the representation of vector hysteresis by the vector Preisach model. $J$. Appl. Phys. (2A) 85, 4379-4381.

[73] Friedman, G., Cha, K., Huang, Y., and Kouvel, J. (2000). Vector form of wipe-out memory and its experimental testing. IEEE Transactions on Magnetics 36, 3185-3188.

[74] Carpenter, J., Dahmen, K., Sethna, J., Friedman, G., Loverde, S., and Vanderveld, A. (2001). Subloops, Barkhausen noise, and disorder induced critical behavior. J. Appl. Phys. 89, 6799-801.

[75] Shukla, P. (2000). Exact Solution of return hysteresis 
loops in a one-dimensional random-field Ising model at zero temperature. Phys. Rev. E 62, 4725-4729.

[76] Dhar, D., Shukla, P., and Sethna, J. (1997). Zerotemperature hysteresis in the random-field Ising model on a Bethe lattice. J. Phys. A 30, 5259-5267.

[77] Colaiori, F., Gabrielli, A., and Zapperi, S. (2002). Rayleigh loops in the random field Ising model on the Bethe lattice. Phys. Rev. B 65, 224404.

[78] Shukla, P. (2001). Exact expressions for minor hysteresis loops in the random field Ising model on a Bethe lattice at zero temperature. Phys. Rev. E 63, 027102/1-4.

[79] Zapperi, S., Magni, A., and Durin, G. (2002). Microscopic foundations of the Rayleigh law of hysteresis. $J$. Magn. Magn. Mat. 242-245P2, 987.

[80] Carpenter, J., Dahmen, K., Mills, A., Weissman, M., Berger, A., and Hellwig, O. (2004). Historyinduced critical phenomena in disordered systems. http://arXiv.org/cond-mat/0401023.

[81] Katzgraber, H., Gary, F., and Zimanyi, G. (2004). Fingerprinting Hysteresis. Physica B 343, 10-14.

[82] Frontera, C., and Vives, E. (2002). Avalanches in the ground state of the 3D Gaussian random field Ising model driven by an external field. Comput. Phys. Commun. 147, 455-458.

[83] Frontera, C., and Vives, E. (2000). Studying avalanches in the ground state of the two-dimensional random field Ising model driven by an external field. Phys. Rev. E 62, 7470-7473.

[84] Carpenter, J., and Dahmen, K. (2003). Barkhausen noise and critical scaling in the demagnetization curve. Phys. Rev. B 67, 020412(R).

[85] Zarand, G., Pazmandi, F., Pal, K., and Zimanyi, G. (2002). Using hysteresis for optimization. Phys. Rev. Lett. 89, 150201.

[86] Berger, A., Pang, A. W., and Hopster, H. (2000). Studies of hysteresis loop criticality in ultrathin Gd(0001)/W(110)-films. J. Mater. Process Manu. 9, 131-142.

[87] Berger, A., Inomata, A., Jiang, J. S., Pearson, J. E., and Bader, S. D. (2000). Experimental observation of disorder-driven hysteresis-loop criticality. Phys. Rev. Lett. 85, 4176-4179.

[88] Berger, A., Inomata, A., Jiang, J. S., Pearson, J. E., Bader, S. D., and Dahmen, K. (2001). Disorder-driven hysteresis-loop criticality in $\mathrm{Co} / \mathrm{CoO}$ films. J. Appl. Phys. 89, 7466-7468.

[89] Berger, A., Pechan, M. J., Compton, R., Jiang, J. S., Pearson, J. E., and Bader, S. D. (2001). Disorder-tuning of hysteresis-loop properties in $\mathrm{Co} / \mathrm{CoO}$-film structures. Physica B 306, 235-239.

[90] Marcos, J., Vives, E., nosa, L. M., Acet, M., Duman, E., Murin, M., Novák, V., and Planes, A. (2003). Disorderinduced critical phenomena in magnetically glassy $\mathrm{Cu}-$ Al-Mn alloys. Phys. Rev. B 67, 224406/1-5.

[91] Kim, D. H., Choe, S. B., and Shin, S. C. (2003). Direct observation of Barkhausen avalanche in Co thin films. Phys. Rev. Lett. 90, 087203/1-4.

[92] Lee, W., Choi, B., Xu, Y., and Bland, J. (1999). Magnetization reversal dynamics in epitaxial Fe/GaAs(001) thin films. Phys. Rev. B 60, 10216-10221.

[93] Schwarz, A., Liebmann, M., Kaiser, U., Wiesendanger, R., Noh, T., and Kim, D. W. (2004). Visualization of the Barkhausen Effect by Magnetic Force Microscopy. Phys. Rev. Lett. 92, 077206/1-4.
[94] Vives, E., and Planes, A. (1994). Avalanches in a fluctuationless first-order phase transition in a random-bond Ising model. Phys. Rev. B 50, 3839-48.

[95] Vives, E., Goicoechea, J., Ortín, J., and Planes, A. (1995). Universality in models for disorder-induced phase transitions. Phys. Rev. E 52, R5-R8.

[96] Zheng, G. (2002). Short-time dynamics of first-order phase transition in a disordered system. J. Phys. A Math. Gen. 35, 10549-10561, and references therein.

[97] Tadic, B. (1996). Nonuniversal Scaling Behavior of Barkhausen Noise. Phys. Rev. Lett. 77, 3843-3846.

[98] daSilveira, R., and Kardar, M. (1999). Critical hysteresis for n-component magnets. Phys. Rev. E 59, 1355.

[99] Vives, E., and Planes, A. (2001). Hysteresis and avalanches in the random anisotropy Ising model. Phys. Rev. B 63, 134431/1-14.

[100] Marchetti, M., and Dahmen, K. (2002). Hysteresis in driven disordered systems: From plastic depinning to magnets. Phys. Rev. B 66, 214201.

[101] Illa, X., Vives, E., and Planes, A. (2002). Metastable random-field Ising model with exchange enhancement: A simple model for exchange bias. Phys. Rev. B 66, 224422 .

[102] Pazmandi, F., Zarand, G., and Zimanyi, G. T. (1999). Self-organized criticality in the hysteresis of the Sherrington-Kirkpatrick model. Phys. Rev. Lett. 83, 1034.

[103] Banerjee, V., and Puri, S. (2001). Hysteresis effects in spin systems with quenched disorder. Phys. Rev. E 63, 026106/1-7.

[104] Borg, R., and Kitchens, T. (1973). Magnetization of Gold-Iron Alloys. J. Phys. Chem. Solids 34, 1323-1334.

[105] Monod, P., Prejean, J., and Tissier, B. (1979). Magnetic hysteresis of CuMn in the spin-glass state. J. Appl. Phys. 50, 7324-7329.

[106] Prejean, J., Joliclerc, M., and Monod, P. (1980). Hysteresis in CuMn - The effect of spin-orbit scattering on the anisotropy in the spin-glass state. J. Phys. (Paris) 41, 427-435.

[107] Chakrabarti, B., and Acharyya, M. (1999). Dynamic transitions and hysteresis. Rev. Mod. Phys. 71, 847.

[108] Gutenberg, B., and Richter, C. F. (1954). Seismicity of the Earth and Associated Phenomena. Princeton University, Princeton, NJ.

[109] Kramer, E., and Lobkovsky, A. (1996). Universal Power Law in the Noise from a Crumpled Elastic Sheet. Phys. Rev. E 53, 1465-1469.

[110] Glanz, J. (June 1, 2000). No Hope of Silencing the Phantom Crinklers of the Opera. New York Times National Desk p. A14.

[111] Burridge, R., and Knopoff, L. (1967). Model and theoretical seismicity. Bull. Seismol. Soc. Am. 57, 34113471.

[112] Rice, J., and Ruina, A. (1983). Stability of steady frictional slipping. J. Appl. Mech. 50, 343.

[113] Carlson, J., and Langer, J. (1989). Mechanical model of an earthquake fault. Phys. Rev. A 40, 6470-6484.

[114] Bak, P., and Tang, C. (1989). Earthquakes as a selforganized critical phenomenon. J. Geophysical Res. 94, 15635-7.

[115] Chen, K., Bak, P., and Obukhov, S. (1991). Selforganized criticality in a crack-propagation model of earthquakes. Phys. Rev. A 43, 625-630.

[116] Olami, Z., Feder, H., and Christensen, K. (1992). Self- 
organized criticality in a continuous, nonconservative cellular automaton modeling earthquakes. Phys. Rev. Lett. 68, 1244-1247.

[117] Miltenberger, P., Sornette, D., and Vanette, C. (1993). Self-Organization and Optimal Random Paths Selected by Spatiotemporal Dynamics of Earthquakes. Phys. Rev. Lett. 71, 3604-3607.

[118] Carlson, J., Langer, J., and Shaw, B. (1994). Dynamics of earthquake faults. Rev. Mod. Phys. 66, 657-70.

[119] Myers, C., Shaw, B., and Langer, J. (1996). Slip complexity in a crustal-plane model of an earthquake fault. Phys. Rev. Lett. 77, 972-975.

[120] Shaw, B., and Rice, J. (2000). Existence of continuum complexity in the elastodynamics of repeated fault ruptures. J. Geophysical Res. 105, 23791-23810.

[121] Ben-Zion, Y., and Rice, J. (1995). Slip patterns and earthquake populations along different classes of faults in elastic solids. J. Geophysical Res. 100, 12959-12983.

[122] Fisher, D., Dahmen, K., Ramanathan, S., and BenZion, Y. (1997). Statistics of Earthquakes in Simple Models of Heterogeneous Faults. Phys. Rev. Lett. 78, 4885-4888.

[123] Fisher, D. (1983). Threshold behavior of charge-density waves pinned by impurities. Phys. Rev. Lett. 50, 14861489.

[124] Fisher, D. (1985). Sliding charge-density waves as a dynamic critical phenomenon. Phys. Rev. B 31, 13961427.

[125] Littlewood, P. (1986). Sliding charge-density waves: a numerical study. Phys. Rev. B 33, 6694-6708.

[126] Narayan, O., and Fisher, D. (1992). Critical behavior of sliding charge-density waves in 4-epsilon dimensions. Phys. Rev. B 46, 11520-11549.

[127] Middleton, A., and Fisher, D. (1993). Critical behavior of charge-density waves below threshold: numerical and scaling analysis. Phys. Rev. B 47, 3530-52.

[128] Myers, C., and Sethna, J. (1993). Collective dynamics in a model of sliding charge-density waves. I. Critical behavior. Phys. Rev. B 47, 11171-11192.

[129] Thorne, R. (1996). Charge-density-wave conductors. Physics Today 49, 42-47.

[130] Bak, P., Tang, C., and Wiesenfeld, K. (1987). Selforganized Criticality: An explanation for $1 / \mathrm{f}$ noise. Phys. Rev. Lett. 59, 381-384.

[131] Bak, P., Tang, C., and Wiesenfeld, K. (1988). Selforganized criticality. Phys. Rev. A 38, 364-374.

[132] deGennes, P. (1966). Superconductivity of Metals and Alloys. Benjamin, New York.

[133] Feynman, R., Leighton, R., and Sands, M. (1963-65). The Feynman Lectures on Physics. Addison Wesley, Redwood City, CA, section 37-3.

[134] Jaeger, H., Liu, C., and Nagel, S. (1989). Relaxation at the Angle of Repose. Phys. Rev. Lett. 62, 40-43.

[135] Nagel, S. (1992). Instabilities in a sandpile. Rev. Mod. Phys. 64, 321-325.

[136] Tewari, S., Schiemann, D., Durian, D., Knobler, C., Langer, S., and Liu, A. (1999). Statistics of shearinduced rearrangements in a two-dimensional model foam. Phys. Rev. E 60, 4385-4396.

[137] Sole, R., and Manrubia, S. (1996). Extinction and selforganized criticality in a model of large-scale evolution. Phys. Rev. E 54, R42-R45.

[138] Newman, M. (1996). Self-organized criticality, evolution, and the fossil extinction record. Proc. R. Soc. Lon- don $B$ 263, 1605-1610.

[139] Newman, M., and Palmer, R. (1999), Models of Extinction: A Review. Http://xxx.lanl.gov/abs/adaporg/9908002.

[140] Cieplak, M., and Robbins, M. (1988). Dynamical Transition in Quasistatic Fluid Invasion in Porous Media. Phys. Rev. Lett. 60, 2042-2045.

[141] Leschhorn, H., Nattermann, T., Stepanow, S., and Tang, L. (1997). Driven interface depinning in a disordered medium. Ann. Phys. (Leipzig) 6, 1-34.

[142] Roters, L., Hucht, A., Lubeck, S., Nowak, U., and Usadel, K. (1999). Depinning transition and thermal fluctuations in the random-field Ising model. Phys. Rev. E 60, 5202-7.

[143] Field, S., Witt, J., Nori, F., and Ling, X. (1995). Superconducting vortex avalanches. Phys. Rev. Lett. 74, 1206-1209.

[144] Ertas, D., and Kardar, M. (1994). Anisotropic Scaling in Depinning of a Flux Line. Phys. Rev. Lett. 73, 17031706.

[145] Ertas, D., and Kardar, M. (1996). Anisotropic scaling in threshold critical dynamics of driven directed lines. Phys. Rev. B 53, 3520-3542.

[146] Lilly, M., Wooters, A., and Hallock, R. (1996). Spatially extended avalanches in a hysteretic capillary condensation system: Superfluid He-4 in nuclepore. Phys. Rev. Lett. 77, 4222-4225.

[147] Guyer, R., and McCall, K. (1996). Capillary condensation, invasion percolation, hysteresis, and discrete memory. Phys. Rev. B 54, 18-21.

[148] Ortin, J., Rafols, I., Carrillo, L., Goicoechea, J., Vives, E., Manosa, L., and Planes, A. (1995). Experiments and models of avalanches in martensites. J. de Physique IV 5, 209-214.

[149] Bouchaud, J. (2005). Power-laws in economy and finance: some ideas from physics. In Beyond Efficiency, proceedings of the Santa Fe conference (J. D. Farmer, and G. Geanakoplos, eds.), Oxford University Press, Oxford, cond-mat/0008103, and references therein, to be published.

[150] Bak, P., Paczuski, M., and Shubik, M. (1997). Price Variations in a Stock Market with Many Agents. Physica A 246, 430-453.

[151] Iori, G., and Jafarey, S. (2001). Criticality in a model of banking crises. Physica A 299, 205-212.

[152] Lu, E., Hamilton, R., McTiernan, J., and Bromond, K. (1993). Solar Flares and Avalanches in Driven Dissipative Systems. Astrophysical Journal 412, 841-852.

[153] Carreras, B., Newman, D., Dobson, I., and Poole, A. (2004). Evidence for self-organized criticality in a time series of electrical power system blackouts. IEEE Transactions on Circuits and Systems I-Regular Papers 51, 1733-1740.

[154] Sachtjen, M., Carreras, B., and Lynch, V. (2000). Disturbances in a power transmission system. Phys. Rev. E 61, 4877-4882.

[155] Watts, D. (2002). A simple model of global cascades on random networks. P. Natl. Acad. Sci. USA 99, 57665771.

[156] Carlson, J., and Doyle, J. (1999). Highly optimized tolerance: A mechanism for power laws in designed systems. Phys. Rev. E 60, 1412-1427.

[157] Carlson, J., and Doyle, J. (2000). Highly optimized tolerance: Robustness and design in complex systems. 
Phys. Rev. Lett. 84, 2529-2532.

[158] Newman, M. (2000). The power of design. Nature 405 , 412-413.

[159] Galam, S. (1997). Rational group decision making: a random field Ising model at $\mathrm{T}=0$. Physica $A$ 238, 6680 .

[160] Alencar, A., Buldyrev, S., Majumdar, A., Stanley, H. E., and Suki, B. (2003). Perimeter growth of a branched structure: Application to cracke sounds in the lung. Phys. Rev. E 68, 011909/1-12.

[161] Petri, A., Paparo, G., Vespignani, A., Alippi, A., and Costantini, M. (1994). Experimental Evidence for Critical Dynamics in Microfracturing Processes. Phys. Rev. Lett. 73, 3423-3426.

[162] Garcimartn, A., Guarino, A., Bellon, L., and Ciliberto, S. (1997). Statistical Properties of Fracture Precursors. Phys. Rev. Lett. 79, 3202-3205.

[163] Curtin, W., and Scher, H. (1991). Analytic model for scaling of breakdown. Phys. Rev. Lett. 67, 2457-2460.

[164] Herrman, H., and Roux, S., (Eds.) (1990). Statistical Models for the Fracture of Disordered Media. North Holland, Amsterdam, and references therein.

[165] Chakrabarti, B., and Benguigui, L. (1997). Statistical Physics of Fracture and Breakdown in Disordered Systems. Clarendon Press, Oxford, and references therein.

[166] Zapperi, S., Ray, P., Stanley, H., and Vespignani, A. (1997). First-Order Transition in the Breakdown of Disordered Media. Phys. Rev. Lett. 78, 1408-1411.

[167] Kadanoff, L. (1966). Scaling Laws for Ising Models near Tc. Physics (Long Island City, NY) 2, 263-272.

[168] Wilson, K. (1979). Problems in physics with many scales of length. Scientific American 241, 140-57.

[169] Pfeuty, P., and Toulouse, G. (1977). Introduction to the Renormalization Group and to Critical Phenomena. Wiley and Sons, London.

[170] Yeomans, J. (1992). Statistical Mechanics of Phase Transitions. Oxford, Oxford.

[171] Fisher, M. (1998). Renormalization group theory: Its basis and formulation in statistical physics. Rev. Mod. Phys. 70, 653-681.

[172] Bruce, A., and Cowley, R. (1981). Structural Phase Transitions. Taylor and Francis, London.

[173] Planes, A., and Manosa, L. (2001). Vibrational properties of shape-memory alloys. SOLID STATE PHYS 55, 159-267.

[174] Vives, E., Ortín, J., nosa, L. M., Ràfols, I., PérezMagrané, R., and Planes, A. (1994). Distributions of avalanches in martensitic transitions. Phys. Rev. Lett. 72, 1694-1697.
[175] Pérez-Reche, F., Stipcich, M., Vives, E., nosa, L. M., Planes, A., and Morin, M. (2004). Kinetics of martensitic transitions in $\mathrm{Cu}-\mathrm{Al}-\mathrm{Mn}$ under thermal cycling: Analysis at multiple length scales. Phys. Rev. B 69, 064101/1-7.

[176] Sreekala, S., and Ananthakrishna, G. (2003). Acoustic Emission and shape memory effect in the martensitic transformation. Phys. Rev. Lett. 90, 135501/1-4.

[177] Ahluwalia, R., and Ananthakrishna, G. (2001). Powerlaw statistics for avalanches in a martensitic transformation. Phys. Rev. Lett. 86, 4076-4079.

[178] Raghavan, V. (1992). Kinetics of Martensitic Transformations. In Martensite (G. Olson, and W. Owen, eds.), ASM International.

[179] Lilly, M., Finley, P., and Hallock, R. (1993). Memory, congruence, and avalanche events in hysteretic capillary condensation. Phys. Rev. Lett. 71, 4186-4189.

[180] Lilly, M., and Hallock, R. (2001). Avalanche behavior in the draining of superfluid helium from the porous material Nuclepore. Phys. Rev. B 64, 024516.

[181] Detcheverry, F., Kierlik, E., Rosinberg, M., and Tarjus, G. (2004). Hysteresis in capillary condensation of gases in disordered porous solids. Physica B 343, 303-313.

[182] Detcheverry, F., Kierlik, E., Rosinberg, M., and Tarjus, G. (2003). Local mean-field study of capillary condensation in silica aerogels. Physical Review E 68, 061504.

[183] Wu, W., and Adams, P. (1995). Avalanches and slow relaxation - Dynamics of ultrathin granular superconducting films in a parallel magnetic field. Phys. Rev. Lett. 74, 610-613.

[184] Blossey, R., Kinoshita, T., and Dupont-Roc, J. (1998). Random-field Ising model for the hysteresis of the prewetting transition on a disordered substrate. Physica A 248, 247-272.

[185] Sabhapandit, S., Dhar, D., and Shukkla, P. (2002). Hysteresis in the random-field Ising model and bootstrap percolation. Phys. Rev. Lett. 88, 197202/1-4.

[186] Durin, G. Invited talk at the Second SPIE International Symposium on Fluctuations and Noise, Maspalomas, Gran Canaria, Spain, May 25-28, 2004.

[187] O'Brien, K., and Weissman, M. (1994). Statistical characterization of Barkhausen noise. Phys. Rev. E 50, 3446-3452.

[188] Petta, J., Weissman, M., and Durin, G. (1998). Barkhausen pulse structure in an amorphous ferrmagnet: Characterization by high-order spectra. Phys. Rev. E 57, 6363-6369. 